\title{
Information and Delay in an Agency Model ${ }^{*}$
}

\author{
Mikhail Drugov ${ }^{\dagger}$
}

May 2006

\begin{abstract}
Negotiations often take long time even if a delay in the agreement is inefficient. One typical explanation is the existence of private information of at least one party; the time is then a discriminating instrument. The paper starts by pointing out that this result does not hold once the traded quantity is not fixed as in most bargaining models; the quantity outperforms the time as a discriminating instrument, that is, there is no delay. Moreover, Coase conjecture does not hold either. We then study how a signal arriving in the course of negotiations affects the delay in the agreement. Unlike investment-under-uncertainty models, a better signal not only improves contracting in the future but also in the present. Therefore, the delay is in general not monotonic in the quality of information. The value of information can be negative over some range as better information may aggravate the principal's commitment problem.
\end{abstract}

JEL Classification: D82, D83, L51.

Keywords: Information, adverse selection, delay, bargaining, Coase conjecture.

\section{Introduction}

Buyer-seller games where a seller sells a good to a buyer whose valuation she does not know have been studied extensively in the past thirty years. There is one important issue that seems to escape the attention of the literature: how some exogenous information affects the trade. In practice, firms and not only them (consumer associations, public agencies, research institutes) do conduct a lot of studies with the aim of understanding

${ }^{*}$ This is the first chapter of my Ph.D. thesis. I am grateful to my advisor Jacques Crémer for support and encouragement. I also benefited from helpful comments from Guido Friebel, Bruno Jullien, Patrick Rey, François Salanié, Jean Tirole and seminar participants of Université de Toulouse, Northwestern University, EEA 2005, EARIE 2005 and JEI 2005. Any remaining errors are my own.

${ }^{\dagger}$ University of Oxford, Nuffield College. E-mail: mikhail.drugov@nuffield.ox.ac.uk 
what consumers value. However, when deciding whether to conduct a market study, and if yes, which, the firm must know the benefits of such a study.

In a very loose way, the trade may be affected in two dimensions in a dynamic setting: first, the amount of trade, and second, its timing. The two, of course, are closely related. For both interpretational and technical reasons we focus on the timing issue. In fact, in bargaining models delaying the trade with the agent serves as a discriminating instrument: the high valuation types are more impatient and are willing to trade earlier. In vast majority of these models the time is the only discriminating instrument available as the size of the traded good is given. Delay arises even in the absence of the exogenous information.

When a signal becomes available in the future, the seller has an additional reason to postpone the trade. A first intuition would suggest that delay can only increase with the quality of the information as in various timing-of-investment models; in the language of Dixit and Pindyck (1994), the option value of waiting increases. In these models, a more precise signal makes investment later more profitable while profits of investing now (before the signal) are constant. In an agency model, if the principal wants to contract with her agent today, she has to give him at least what he can get tomorrow from the relationship. Then, better information tomorrow decreases the agent's tomorrow rent and so, improves contracting today. The delay decreases with the quality of information.

We use a very simple two-period two-type setup. For modelling reasons, we chose a production formulation where an agent produces some good of a given size for the principal; the agent is privately informed about his costs. ${ }^{1,2}$ In the first period the principal posts an offer, which is a price that she is ready to pay for the good. As typical in bargaining models, the offer is valid only the current period and the principal cannot commit to anything in the second period. The agent can accept the offer in which case the production and the transfer take place in the first period and the game is over. If he refuses, the game moves to the second period. They both observe a signal that provides some information about the agent's type. The principal makes an offer that the agent is free to accept or reject.

We concentrate on the timing of production. Knowing the timing of production by different types it is very easy to reconstruct the corresponding offers of the principal and the resulting rents of the two players. In particular, we are interested in the delay, i.e., how often production takes place in the second period when it could be done in the first

\footnotetext{
${ }^{1}$ The reason is that when the quantity is variable in the buyer-seller formulation there is always a possibility that the low valuation type is not served. In the production formulation, the inefficient type is never shut down provided that some conditions on the principal's surplus function are satisfied.

${ }^{2}$ The low cost type in the production formulation corresponds to the high valuation type in the buyerseller formulation, and the high cost type corresponds to the low valuation type.
} 
period. We focus on the equilibrium where the principal posts the low cost offer in both periods and the low cost agent plays a mixing strategy (accepting the first-period offer with some probability) such that the principal is indifferent which offer to make in the second period. The high cost agent does not produce at all; in this case the delay is the probability that the low cost type produces in the second period. The standard result is that there is delay even in the absence of future information.

As a first step, consider a perfect signal about the agent with probabilities conditional on the agent's type, $\underline{p}$ and $\bar{p}$ for the low and high cost type, respectively. In other words, if the agent is low cost there is a signal that reveals his type with probability $\underline{p}$ and no signal with probability $1-\underline{p}$; if the agent is high cost the probability of the signal is $\bar{p}$. Then, I will show that the delay increases with $\underline{p}$ and decreases with $\bar{p}$. A higher $\underline{p}$ makes the principal assign a higher weight to the high cost type if there is no signal in the second period. The low cost type has to delay his production more often to keep the principal indifferent in the second period with respect to the offer she makes. For $\bar{p}$, the logic is reversed.

This example, and more generally, any signal with a discreet support is rather specific: the mixing probability of the low cost type is determined in the way to keep the principal indifferent. With a continuous (atomless) support of the signal the picture changes. After any realization of the signal the principal prefers strictly either a low or a high offer. The low cost type then earns a rent in the second period every time the principal makes a high offer to a low cost agent. To keep the low cost agent mixing between the two periods the principal has to leave him exactly the same rent in the first period. The optimal mixing probability, and therefore, delay, are determined by considerations at the margin. The fixed size of the good implies that the "intensity" of beliefs does not matter: the offer of the principal and consequently, the agent's rent depend on whether principal's beliefs pass some threshold but do not depend on how much they pass it. Then, only probabilities at a certain threshold enter the expression. Changing the distribution in any way without touching a little interval at the threshold does not affect delay.

The discussion above reveals that a model with a fixed size of the good (call it the "0-1" model in the paper) may have some particular features that do not survive in a richer framework. To address this issue, we consider a "variable quantity" model where the production has a quantity dimension. This extension does make important changes. In a benchmark case, when there is no signal, there is no delay: the principal contracts with the agent as if the second period does not exist. This is what the principal would do, had he the commitment power. This is an important result for three reasons: first, it stands in a drastic contrast with the standard "0-1" model; second, it shows that in this model the no-commitment solution coincides with the full commitment one; third, it proves that the principal prefers to use the quantity, and not time, to discriminate among 
different types of the agent. It also goes against the Coase conjecture that says that the non-ability of the monopolist to commit to long-term offers destroys his monopoly rent. Unfortunately, on the very sad day of the 30th of March, 2006, I discovered a paper by Wang (1998) that shows exactly the same result in the same setting, though, with the help of a much longer and more complicated proof than mine.

When it comes to a signal, its support, discreet or continuous, does not matter much in a model with variable quantity. It is shown that there is either no delay (all production takes place in the first period) or the high cost type produces only in the second period and the low cost type mixes, as in "0-1" model, between production in the first and in the second periods. The delay in this case is the production by the low cost type in the second period. With variable quantity at hand, the principal proposes a different BaronMyerson contract for any possible beliefs in the second period. The expected rent of the low cost type depends on the precise shape of the distribution of the signal. Therefore, any change in the distribution affects the optimal delay. Moreover, the marginal rent that determines the optimal delay, might be affected in a non-monotonic way by any change

in the distribution. Consequently, any results such as "better information increases (or decreases) delay" might not be valid without further assumptions.

The rest of the paper is organized as follows. The benchmark case, when there is no signals, is treated in Section 2, in the "0-1" model and then in the "variable quantity" model. Section 3 studies the effects of signals on delay: a perfect signal in the "0-1" model (Section 3.2); a signal with a continuous support in the "0-1" model (Section 3.3); a general case of the "variable quantity" model (Section 3.4); and finally, the same perfect signal as before but in the "variable quantity" model (Section 3.5). Section 4 concludes. Some proofs are given in text, some are contained in Appendix B.

\section{The Benchmark: no signals}

In this section we study the benchmark case when there are no signals. Thus, the principal does not want to delay her contracting with the agent to learn some new information about the agent. However, she still might postpone dealing with the agent as a means of discrimination among different agent's types.

The dynamic bargaining models (with one-sided offers) are typically done in the sellerbuyer context, i.e., when a seller of a certain good proposes a price to a buyer whose valuation she does not know. See Fudenberg and Tirole (1983), Sobel and Takahashi (1983) and many others. We prefer the production interpretation, like in Wang (1998), where the agent with unknown costs produces a good for the principal. The reason is the following: when the quantity is variable, in the buyer-seller model there is always shutdown for low valuation types if their share is small enough. In the production formulation, 
some conditions on the principal's surplus function guarantee that the shutdown policy is never profitable for any distribution of types. This makes the analysis more tractable. In economic terms, the results obtained in one formulation can be easily interpreted in the spirit of another one.

\section{$2.1 \quad$ "0-1" Model}

Except for the buyer-seller formulation, the setup and analysis of the "0-1" model follows quite closely the textbook version of Bolton and Dewatripont (2005), ch. 9.1, who, in their turn, follow Hart and Tirole (1988). Their model as well several previous papers (such as Stokey (1981)) consider durable goods; however, some such as Gul, Sonnenschein, and Wilson (1986) consider a good consumed in one period. We prefer non-durable formulation which is equivalent to a durable one in a two-period model like ours. ${ }^{3}$

There are two periods. The principal needs at most one unit of the good to be produced by the agent, i.e., the agent produces one unit in either the first period or the second one or does not produce at all. The principal derives surplus $S$ from the good . The agent has costs of production $\theta$ which are his private information. There are two types of agents: a low cost agent has costs $\underline{\theta}$ and a high cost agent has $\operatorname{costs} \bar{\theta}$ with $\Delta \theta \equiv \bar{\theta}-\underline{\theta}>0$. Both types are socially efficient: $\underline{\theta}<\bar{\theta}<S$. The type is the same in the two periods. The initial probability of a low cost type $\underline{\theta}$ is $\nu$. There is a common discount factor $\delta<1$.

When production takes place in the first period, the principal gets profits of $S-t$ while the agent gets a rent of $t-\theta$, where $t$ is a monetary transfer from the principal to the agent. If production happens in the second period, both utilities are multiplied by the discount factor $\delta<1$. Outside option for all the players is zero.

We are looking for a Perfect Bayesian equilibrium (PBE) of this game. As typical for dynamic agency models, sometimes there are many PBEs. We focus on the one that gives the maximum payoff for the principal (sometimes we call it optimal for the clarity of exposition). This is the standard way to proceed in the literature, see, in particular, Laffont and Tirole (1987); Laffont and Tirole (1990) for adverse selection environment and Fudenberg and Tirole (1990) for the moral hazard setting, Bester and Strausz (2001) for a version of revelation principle in adverse selection environment with imperfect commitment. In this basic formulation the game is well known, thus, we will only state the results without proving them.

\subsubsection{Full commitment}

Assume that the principal has a commitment power. Then she faces two alternatives: either to pay a high first-period transfer $t_{1}=\bar{\theta}$ and have both types producing in the

\footnotetext{
${ }^{3}$ With a proper redefinition of the discount factor.
} 
first period or commit to a low transfer $t_{1}=t_{2}=\underline{\theta}$ and have only the low cost agent producing. Define the threshold $\nu^{1}$ as follows:

$$
\nu^{1}=\frac{S-\bar{\theta}}{S-\underline{\theta}}
$$

Then, the optimal principal's policy is characterized in Proposition 1.

Proposition 1 Under full commitment, if $\nu<\nu^{1}$, the principal sets $t_{1}=\bar{\theta}$ and both types produce in the first period. If $\nu \geq \nu^{1}$, the principals sets $t_{1}=t_{2}=\underline{\theta}$; the low cost agent produces in the first period, the high cost agent does not produce at all.

\subsubsection{No commitment}

We now examine the case when the principal cannot commit to a long-term contract. Without loss of generality, the game is as follows: in the first period the principal announces a price $t_{1}$ that she will pay to the agent if he produces in that period. If the agent accepts, the production takes place, the transfer is paid and the game ends. If the agent refuses, they go to the second period and the principal makes another proposal $t_{2}$; if he refuses in the second period, the game ends and each party gets zero.

Proposition 2 There are two thresholds $\nu^{1}$ (defined by (1)) and $\nu^{2}, 0<\nu^{1}<\nu^{2}<1$, such that

Case 1. If $\nu<\nu^{1}$, the principal sets the first-period transfer at $t_{1}=\bar{\theta}$ and both types produce in the first period.

Case 2. If $\nu^{1} \leq \nu<\nu^{2}$, the principal sets the first-period transfer at $t_{1}=\underline{\theta}+\delta \Delta \theta$ and the low cost agent produces in the first period. She sets the second-period transfer at $t_{2}=\bar{\theta}$ and the high cost agent produces in the second period.

Case 3. If $\nu \geq \nu^{2}$, the principal sets both transfers at $t_{1}=t_{2}=\underline{\theta}$. The low cost agent produces with probability

$$
\gamma=1-\frac{\nu^{1}}{1-\nu^{1}} \frac{1-\nu}{\nu}
$$

in the first period and with probability $1-\gamma$ in the second period. The high cost agent does not produce at all.

\footnotetext{
${ }^{4}$ It equals

$$
\nu^{2}=\frac{(1-\delta) \frac{\nu^{1}}{1-\nu^{1}}+\delta \nu^{1}}{(1-\delta) \frac{\nu^{1}}{1-\nu^{1}}+\delta}
$$
}


When the low cost agent is very unlikely to be dealt with, the principal prefers to pay a high transfer and to have all the production in the first period. When it becomes more likely that the agent is low cost, the principal distorts the high cost agent production by moving it to the second period and decreases the rent of the low cost agent. When the low cost agent becomes even more likely, the principal sacrifices the production of the high cost agent in order not to pay any rent to the low cost agent. However, inability of the principal to commit not to contract with the high cost agent in the second period makes it necessary to have the low cost agent in the second period with some probability. More precisely, the mixing probability $\gamma$ is such that the second-period probability of the low cost agent is exactly $\nu^{1}$ at which the principal is indifferent between setting the transfer at $\underline{\theta}$ or $\bar{\theta}$.

Comparing Propositions 1 and 2 we see that under no commitment when probability of the low cost agent is higher than the threshold $\nu^{1}$ it becomes impossible for the principal to stick to her full commitment solution. The reason is that after the first-period low price was rejected by the agent, the principal wants to increase the offer as she thinks that the agent is high cost. But then the low cost agent would reject the first-period low price. The principal has two solutions: either to pay a rent to the low cost agent in the first period or to have the low cost agent in the second period with some probability as a commitment against increasing the second-period offer.

\section{2 "Variable quantity" Model}

In this section we keep the same model but we assume that the quantity may vary. The principal needs the agent to produce only once, either in the first period or in the second period. Denote by $q$ the quantity produced by the agent. The principal's surplus is now a function $S(q)$ where $q$ is the quantity or the quality of the production. The function $S$ is concave and satisfies the Inada conditions: $S^{\prime}(q)>0, S^{\prime \prime}(q)<0, S(0)=0$ and $S^{\prime}(0)=\infty .^{5}$ The agent has linear costs of production $C(q)=\theta q$ where $\theta$ is his private information as before.

\subsubsection{Full commitment}

When the principal can commit to two-period offers, she does not want to delay the production: if there is any quantity produced in the second period by some type, the principal would propose to this type to produce this quantity times the discount factor in the first period and to receive the associated transfer times the discount factor also in the same period. Since the agent's utility is linear, both types would be indifferent

\footnotetext{
${ }^{5}$ Later (Assumption 2 on p. 29) we also assume that $S^{\prime \prime \prime}(q)>0$.
} 
to this change. The principal would gain as the surplus function $S(q)$ is concave. Thus, she solves the standard (one-period) Baron-Myerson problem (see Baron and Myerson (1982) for the original paper and Laffont and Martimort (2002), ch. 2, for a textbook exposition).

Introduce the standard notation by replacing transfers by rents: $\bar{U}_{\tau} \equiv t_{\tau}-\bar{\theta} q_{\tau}$ and $\underline{U}_{\tau} \equiv t_{\tau}-\underline{\theta} q_{\tau}$ where index $\tau$ stands for the period.

Proposition 3 Under full commitment, the agent produces in the first period according to the standard Baron-Myerson contract $\left\{\left(\underline{q}_{1}, \underline{U}_{1}\right),\left(\bar{q}_{1}, \bar{U}_{1}\right)\right\}$ :

- The low cost agent produces at the first-best level, $\underline{q}_{1}=\underline{q}^{F B}$, given by $S^{\prime}\left(\underline{q}^{F B}\right)=\underline{\theta}$, and earns a positive rent, $\underline{U}_{1}=\Delta \theta \bar{q}_{1}$.

- The high cost agent's quantity is distorted downwards, $\bar{q}_{1}=\bar{q}^{S B}$, given by $S^{\prime}\left(\bar{q}^{S B}\right)=$ $\bar{\theta}+\frac{\nu}{1-\nu} \Delta \theta$, and his rent is zero, $\bar{U}_{1}=0$.

The principal commits to the null contract in the second period.

Proof. Under full commitment, apply the Revelation Principle. The agent truthfully reports his type and receives one of the two pre-specified contracts. In full generality these contracts may be stochastic. Let us show that any production in the second period cannot be part of an optimal contract. Assume that the contract for some type specifies some production in the second period and the corresponding transfer $\left(q_{2}, t_{2}\right)$ either with probability one if the contract is deterministic or with some probability if the contract is stochastic. The principal is better off if she replaces $\left(q_{2}, t_{2}\right)$ by $\left(\delta q_{2}, \delta t_{2}\right)$ to be produced and paid in the first period with the same probability as $\left(q_{2}, t_{2}\right)$ since the surplus function $S(q)$ is concave. The agent's rent and so his individual rationality and incentive compatibility constraints are not affected as his utility is linear in the quantity and the transfer. Thus, all production takes place in the first period.

Then, the principal solves the standard Baron-Myerson problem as if the second period does not exist. The optimal contract takes the form stated in Proposition. The commitment of the principal to the null contract assures that no type wants to deviate and to refuse the first-period offer.

\subsubsection{No commitment}

Very recently (the sad day of the 30th of March) I discovered the paper of Wang (1998) that makes exactly the same point as this Section (Proposition 4), in exactly the same setting. I continue with my original proof and discuss the differences and similarities at the end of this Section. 
Is the optimal contract different once the principal does not have the commitment power? We have seen that with "0-1" quantity no-commitment optimal contract differs from the full commitment one when the probability of the low cost type is high enough. The principal proposes a low price for the production in the first period and if the offer is rejected, she cannot commit not to increase the price in the second period to contract with the high cost type. Thus, some kind of distortion was needed to assure that the low cost type does not always reject the first-period offer to get a better deal later.

When the quantity can vary, the situation is different. The principal can deal with both types in the first period on different terms. Then, if the agent refuses the offer, the principal might not become pessimistic about the agent's type and might not make a better offer in the second period. In this case, the low cost type will not reject the firstperiod offer. In fact, as the next Proposition shows, the principal is able to implement the full commitment solution.

Proposition 4 Under no commitment, the principal implements the full commitment solution given in Proposition 3.

Proof. The principal's payoff under no commitment is bounded by his full commitment payoff. Thus, if we show that the principal is able to implement the full commitment solution under no commitment, the problem is solved.

Let us look at the following candidate PBE: the principal proposes the full commitment menu (specified in Proposition 3) in the first period and the agent accepts it (choosing the contract corresponding to his type). The principal gets the full commitment payoff. To complete the characterization of this PBE, one has to specify what happens if the agent rejects the first-period offer.

Since the agent is supposed to accept the first-period offer, a rejection is on the offequilibrium path. The principal cannot revise her beliefs according to the Bayes rule and there is some freedom in assigning her off-equilibrium beliefs. Assume that she thinks that both types are equally likely to deviate. Then, in the second period she has the same beliefs as in the first period and offers the same menu as in the first period. The low cost type is strictly worse off if he deviates as his rent is discounted. The high cost type is indifferent since his rent is always zero. Thus, this is an equilibrium.

Remark 1. In this PBE with such out-of-equilibrium beliefs the low cost type is strictly worse off by deviating while the high cost type always gets zero. One may think that "reasonable" out-of-equilibrium beliefs should assign weight 1 to the high cost type. Let us argue why it is not exactly the case.

Let us start by noting that if $\delta \leq \frac{\bar{q}^{S B}}{\bar{q}^{F B}}$, where $\bar{q}^{S B}$ is given by Proposition 3 and $\bar{q}^{F B}$ is given by $S^{\prime}\left(\bar{q}^{F B}\right)=\bar{\theta}$, then the out-of-equilibrium beliefs are of no importance. The low 
cost type in the best case would get the second period rent of $\delta \Delta \theta \bar{q}^{F B}$ which is not more than his first period rent of $\Delta \theta \bar{q}^{S B}$.

The case of interest is, therefore, when $\delta>\frac{\bar{q}^{S B}}{\bar{q}^{F B}}$. Then, the deviation by the low cost type is not dominated in the following sense: his equilibrium payoff of $\Delta \theta \bar{q}^{S B}$ might be smaller than his deviation payoff for some principal's out-of-equilibrium beliefs (the ones that assign enough weight to the high cost agent). His deviation is not unreasonable.

Another argument is that one can assume that there exist arbitrarily small positive costs of going to the second period borne by the agent. The principal might propose the reimbursement of these costs when she wants. The full commitment equilibria in both "0-1" and variable quantity models would be unchanged. In the equilibrium of "0-1" model with no commitment the principal would propose to reimburse these costs to the agent should the agent reject the first-period offer; the structure of equilibrium is then unchanged. In the present case these costs will make the high cost type strictly prefer to produce in the first period. In fact, he prefers to leave the principal than to go to the second period. Then, observing a rejection of the first-period offer and the fact that the agent is still in the relationship should convince the principal that the agent is low cost.

The proof of Wang (1998) is much longer, more complicated and less general. In particular, his proof cannot be easily generalized to the continuum of agent's types while the logic of my proof does not depend at all on the number of types. His model has an infinite horizon but it can be incorporated in my model without any change in the proof. He also runs in the same problem of out-of-equilibrium beliefs but he only briefly comments on it (ft. 5).

This result should be contrasted with the one of Bagnoli, Salant, and Swierzbinski (1995) where the setting is very similar. They have a buyer-seller game where the seller chooses the quality of the good and can propose menus of quality-price pairs. The difference is that there are two buyers with high and low valuation. There, even with full commitment, there is a delay. The seller can sell to both agents in the first period with the standard Baron-Myerson contract but she prefers to do the following (if the discount factor is sufficiently close to one): she sells to the high valuation type his first-best quality good leaving him no rent and then does the same to the low valuation type in the second period. The high valuation type does not want to deviate since if he deviates, the seller knows that there is the high valuation type left and proposes again the no-rent contract for this type. As the quality is never distorted in their model, the same outcome would be if the quantity is fixed. The authors conclude that "Inducing self-selection over time turns out in this model to dominate inducing self-selection at a point in time", i.e., a completely opposite result from the one in our model where separation occurs only at a point in time (in the first period). In the companion working paper (that I could not 
find) Bagnoli, Salant, and Swierzbinski (1992) show the same results when the seller is uncertain about the realized distribution of buyer types provided buyer types are correlated. The correlation is crucial as observing the agents that accept an offer provides some information about those that have not yet accepted.

\section{Signals}

In this Section we introduce a signal that arrives between the two periods and provides some information about the agent's type. Thus, the principal has an incentive to postpone the contracting with the agent, to obtain a signal and to contract with the agent in the second period at better terms. We study how this waiting, or delay, depends on the characteristics of the signal. Note that in "0-1" model when $\nu \geq \nu^{1}$ delay occurs even in the absence of the signal while in the variable quantity model there is no delay where there is no signal. When there is no commitment, the offers cannot be made conditional on the realization of the signal. The first-period contracts are enforced before the signal becomes available.

The signal is a random variable with the cumulative distribution function $F$ and the density function $f$. When the signal is realized and takes some value $i$ (a bit loosely we will often say "signal $i$ " for a particular realization of the signal and "signal" for the whole distribution $)^{6}$, denote by $f_{i}(k)$ the conditional probability that the agent is of type $k \in\{l, h\}$ ( $k=l$ for "low cost" or $h$ for "high cost"). For each signal $i$ the principal's updated beliefs in the second period are $f_{i}(l)$ and $f_{i}(h)$ for the low and high cost agent, respectively. However, it will be useful to characterize the distribution of the signal by the conditional probabilities of signal $i$ given that the agent is of type $k, f_{k}(i)$. There are two reasons why we prefer the characterization of the signal by probabilities $f_{k}(i)$. First, these probabilities do not depend on the initial prior $\nu$ and the strategy of the agent in the first period (for example, on the mixing probability $\gamma$ in the "0-1" model). Second, it is these probabilities that enter into calculation of the expected second-period rent from the agent's point of view. Indeed, the agent knows his type, thus when he decides whether to accept a first-period offer with some rent he computes his expected rent if he goes to the second period using these conditional probabilities.

When the support of the signal's distribution is finite, denote $f_{i}(k) \equiv p_{k \mid i}$ and $f_{k}(i) \equiv$ $p_{i \mid k}$. It is useful to represent this signal as following matrix:

\footnotetext{
${ }^{6}$ In fact, " $i$ " is an index of the signal, any renumbering or renaming of signal's realizations does change the signal.
} 


$$
\left(\begin{array}{cc}
p_{1 \mid l} & p_{1 \mid h} \\
\vdots & \vdots \\
p_{n \mid l} & p_{n \mid h}
\end{array}\right) \quad \begin{gathered}
\leftarrow \text { signal } 1 \\
\longleftarrow \text { signal } n
\end{gathered}
$$

In this matrix a row $i$ consists of two probabilities $p_{i \mid l}$ and $p_{i \mid h}$ for signal $i$. Thus, an element $(i, k)$ is the conditional probability of the realization $i$ on the agent being of type $k$. Note that the sum of elements of each column equals one.

\subsection{The general case of the "0-1" model}

In this Section we show that for any signal there are only three strategies of the agent that can be part of an optimal PBE. There are the same as in case of no signal (Proposition $2)$.

Proposition 5 For any signal, in an optimal PBE the agent does one of the following:

Case 1. Both types produce in the first period, or

Case 2. The low cost type produces in the first period and the high cost type produces in the second period, or

Case 3. The low cost type mixes between producing in the first and in the second period, and the high cost type does not produce at all or produces in the second period depending on the realization of the signal.

Proof. We saw in Proposition 2 that each of the three cases can be optimal.

We have to show that no other case is possible. The number of potential cases is finite; for all of them the proof is very straightforward. We show how it is done for one case: that the high cost never produces in both periods.

Assume the opposite. The principal proposes $t_{2}=\bar{\theta}$ since he cannot commit to propose anything higher and the high cost agent would not produce for less; $t_{1}$ must be also equal $\bar{\theta}$ to make the high cost type indifferent. The low cost type gets the rent of $\Delta \theta$ if he produces in the first period and at most $\delta \Delta \theta$ if he produces in the second period. Then, he produces only in the first period. The principal gains by moving all the production of the high cost type to the first period (for example, proposing $t_{1}=\bar{\theta}+\epsilon$ ).

This Proposition will allow us to focus on only these three cases when studying different signals without proving each time that no other case is optimal. 


\subsection{Example of a signal in the "0-1" model}

Call a particular realization $i$ of a signal perfect if either $p_{l \mid i}$ or $p_{h \mid i}$ is zero. Observing such a realization the principal knows the type of her agent.

Consider a very simple signal. With some probability conditional on the agent's type the realization of the signal is perfect and with the complimentary probability there is no revelation, i.e., there are three realizations of the signal \{low cost, high cost, $\varnothing$ \}. Let $\underline{p}$ be the conditional probability of the perfect realization of the signal if the agent is low cost and $\bar{p}$ if he is high cost. The signal can be represented as

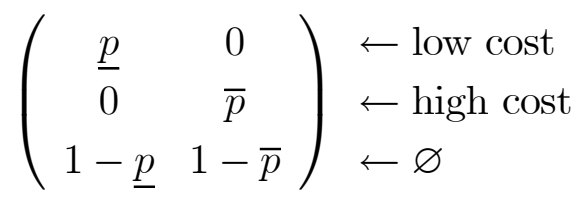

Under full commitment, the principal can make the highest offer $t_{1}=\bar{\theta}$ and earn

$$
V_{F}^{1}=S-\bar{\theta}
$$

She can alternatively focus on the low cost type and set both transfers at low level. But if in the second period she receives the perfect signal that the agent is high cost, she increases the transfer. Thus, her profits $\operatorname{are}^{7}$

$$
V_{F}^{2}=\nu(S-\underline{\theta})+\delta(1-\nu) \bar{p}(S-\bar{\theta}) .
$$

The profits increase with $\bar{p}$ and so this offer structure becomes more profitable than the sure production in the first period for a larger range of parameters.

Under no commitment, there are two cases. First, $\underline{p}$ and/or $\bar{p}$ equals one. In this case, in the second period the principal always knows the type of her agent. Then, she either makes a high offer in the first period $t_{1}=\bar{\theta}$ (both types produce in the first period) or she makes a low offer $t_{1}=\underline{\theta}$ in the first period and the second-period offer high or low depending on the signal (the low cost type produces in the first period and the high cost type produces in the second period). Second, neither $\underline{p}$ nor $\bar{p}$ equals one. The structure of equilibria is then the same as with no signal, i.e., $\underline{p}=\bar{p}=0$. Proposition 2 can be applied with modified thresholds $\nu^{1}$ and $\nu^{2}$, transfers $t_{1}$ and $t_{2}$ and the mixing probability $\gamma$ which are now functions of $\underline{p}$ and $\bar{p}$. Next Proposition characterizes the PBE in more details.

Proposition 6 If $\underline{p}$ and/or $\bar{p}$ equals one, there is a threshold $\nu^{0}=\frac{(1-\delta) \nu^{1}}{1-\delta \nu^{1}}$ such that

\footnotetext{
${ }^{7}$ It is crucial that the signal is perfect. If there is always some noise, the full commitment solution does not change.
} 
- If $v<\nu^{0}$, the principal makes a high first-period offer $t_{1}=\bar{\theta}$ and both types produce in the first period.

- If $v \geq \nu^{0}$, the principal makes a low first-period offer $t_{1}=\underline{\theta}$. The low cost type produces in the first period and the high cost type produces in the second period.

If neither $\underline{p}$ nor $\bar{p}$ equals one, there are two thresholds ${ }^{8} \bar{\nu}^{1}$ and $\nu^{2}, 0<\bar{\nu}^{1}<\bar{\nu}^{2}<1$, such that

Case 1. If $\nu<\bar{\nu}^{1}$, the principal makes a high first-period offer $t_{1}=\bar{\theta}$ and both types produce in the first period.

Case 2. If $\bar{\nu}^{1} \leq \nu<\bar{\nu}^{2}$, the principal makes the first-period offer $t_{1}=\underline{\theta}+\delta(1-\underline{p}) \Delta \theta$ and the low cost agent produces in the first period. She sets the second-period transfer at $t_{2}=\bar{\theta}$ and the high cost agent produces in the second period. Her payoff is

$$
V_{N}^{2}=[\nu+(1-\nu) \delta] S-\nu \underline{\theta}-\delta \bar{\theta}+\delta \nu[\underline{p} \bar{\theta}+(1-\underline{p}) \underline{\theta}] .
$$

Case 3. If $\nu \geq \bar{\nu}^{2}$, the principal makes a low first-period offer $t_{1}=\underline{\theta}$. The low cost agent produces with probability

$$
\gamma=1-\frac{\nu^{1}}{1-\nu^{1}} \frac{1-\nu}{\nu} \frac{1-\bar{p}}{1-\underline{p}} .
$$

in the first period and with the complimentary probability he produces in the second period. The high cost agent produces in the second period with probability $\bar{p}$. The principal's payoff is

$$
V_{N}^{3}=\nu[\gamma+(1-\gamma) \delta](S-\underline{\theta})+\delta(1-\nu) \bar{p}(S-\bar{\theta})
$$

Proof. Proposition 5 gives the three possible cases we should consider.

If $\underline{p}$ and/or $\bar{p}$ equals one, the type of the agent is known in the second period. The principal chooses between the sure production in the first period with the payoff $S-\bar{\theta}$ and delaying the high cost type to save on the low cost type rent, her payoff is

\footnotetext{
${ }^{8}$ These thresholds are

$$
\begin{aligned}
\bar{\nu}^{1} & =\frac{(1-\delta) \nu^{1}}{1-\delta+\delta \underline{p}\left(1-\nu^{1}\right)}<\nu^{1}, \text { and } \\
\bar{\nu}^{2} & =\frac{(1-\delta) \frac{\nu^{1}}{1-\nu^{1}} \frac{1-\bar{p}}{1-\underline{p}}+\delta \nu^{1}(1-\bar{p})}{(1-\delta) \frac{\nu^{1}}{1-\nu^{1}} \frac{1-\bar{p}}{1-\underline{p}}+\delta \nu^{1}(1-\bar{p})+\delta\left(1-\nu^{1}\right)(1-\underline{p})}<1 .
\end{aligned}
$$
}




$$
[\nu+(1-\nu) \delta] S-\nu \underline{\theta}-\delta(1-\nu) \bar{\theta} .
$$

Comparing the two payoffs, gives the threshold $v^{0}$.

If neither $\underline{p}$ nor $\bar{p}$ equals one, the structure of equilibria is the same as with no signal (Proposition 2).

Case 1. The principal sets the first-period transfer at high level: $t_{1}=\bar{\theta}$ and production takes place in the first period. Her payoff $V_{N}^{1}$ is

$$
V_{N}^{1}=S-\bar{\theta}
$$

Case 2. The principal sets the second-period transfer at the high level unless she receives a signal that the agent is low cost. Thus, if the low cost type goes to the second period, his expected rent is $\underline{U}_{2}=\delta(1-\underline{p}) \Delta \theta$. Note that we take the expectation of the rent from the point of view of the low cost agent, i.e., we use conditional probabilities $p_{i \mid l}$. To induce the low cost agent to produce in the first period the principal should provide him at least the same rent, thus, she sets $t_{1}=\underline{\theta}+\delta(1-\underline{p}) \Delta \theta$ and her profits are

$$
V_{N}^{2}=[\nu+(1-\nu) \delta] S-\nu \underline{\theta}-\delta \bar{\theta}+\delta \nu[\underline{p} \bar{\theta}+(1-\underline{p}) \underline{\theta}] .
$$

The probability of the perfect realization conditional of the agent being low cost $p$ increases the principal's profits since it decreases the agent's rent. The probability $\bar{p}$ does not affect the profits since it does not affect the low cost type's rent: neither the probability to get it $(1-\underline{p})$ nor its size $\Delta \theta$ depend on $\bar{p}$.

Case 3. The principal sets both transfers at low level $t_{1}=t_{2}=\underline{\theta}$ and the low cost agent produces in the first and in the second period with some probabilities $\gamma$ and $1-\gamma$. He does not receive any rent. If the agent is high cost he produces in the second period after the perfect realization. The principal's payoff is (5). For this to be an equilibrium the principal has to be indifferent in the second period with respect to the transfer she sets. The second-period principal's beliefs, $\nu_{2}(\gamma)$, must equal the threshold level $\nu^{1}$ defined in $(1)$ :

$$
\frac{\nu(1-\gamma)(1-\underline{p})}{\nu(1-\gamma)(1-\underline{p})+(1-\nu)(1-\bar{p})}=\nu^{1} .
$$

We then obtain the expression for $\gamma(4)$.

Let us see Case 3 in more detail. How does the mixing probability $\gamma$ change with respect to $\underline{p}$ and $\bar{p}$ ? Here, delay is the probability of production in the second period $1-\gamma$. Inspecting (4), we have the following Proposition. 
Proposition 7 Take the signal described by (3). Assume that the principal makes the low cost type randomize between accepting the low transfer in the first and in the second period $\left(\nu \geq \nu^{2}\right)$. Then, the delay is decreasing in $\bar{p}$ and increasing in $\underline{p}$.

A higher $\bar{p}$ means that in the second period in case of no revelation the principal puts more weight on the low cost type. Then, to keep her indifferent between setting the high and the low transfer the low cost type should accept the first-period offer more often, i.e., delay decreases. The effect of a higher $p$ is exactly the opposite. Note that a higher $\underline{p}$ or $\bar{p}$ improves the signal. Thus, Proposition 7 tells us that the delay may increase or decrease with an improved information depending on the precise way the improvement is introduced.

Let us now analyze the effect of an improved signal on principal's profits (5):

$$
\begin{aligned}
& \frac{\partial V_{N}^{3}}{\partial p}=\nu(1-\delta)(S-\underline{\theta}) \frac{\partial \gamma}{\partial \underline{p}}<0, \\
& \frac{\partial V_{N}^{3}}{\partial \bar{p}}=\nu(1-\delta)(S-\underline{\theta}) \frac{\partial \bar{\gamma}}{\partial \bar{p}}+\delta(1-\nu)(S-\bar{\theta})>0 .
\end{aligned}
$$

The negative effect of higher $\underline{p}$ on profits is surprising. A better information decreases profits. The reason is inability of the principal to commit: a higher $\underline{p}$ makes production in the first period less frequent to keep the principal's indifference in the second period. Because of discounting, principal looses. The positive effect of a higher $\bar{p}$ has two parts: first, through the change in the mixing probability $\gamma$; second, a direct effect of producing at high cost when the agent is indeed high cost where otherwise there would be no production.

\subsection{Continuous signal in the "0-1" model}

Let us consider now a more realistic case. There is some noise so any realization of the signal is possible. Realizations of the signal are indexed by $i$; the signal has the cumulative distribution function $F(i)$ and the density function $f(i)$ on the range $[0,+\infty]$. The distribution is atomless. Without loss of generality make the following normalization: $\frac{f_{l}(i)}{f_{h}(i)} \equiv i$. A higher $i$ corresponds to a higher probability of the low cost type. Denote by $F_{k}(i)$ the cumulative probability of realizations up to $i$ when the agent is of type $k$.

As the signal is atomless, if there is one type that is supposed to be in the second period in some equilibrium, a signal assuring the principal of the opposite has probability of measure zero. ${ }^{9}$ For example, if in an equilibrium the low cost agent is supposed to accept the first-period offer and he rejects it mimicking the high-cost agent, the probability of a realization such that the principal understands that there was a deviation (not being able to update her beliefs by Bayes rule) is of measure zero. Thus, we have the following Lemma.

\footnotetext{
${ }^{9}$ In fact, the condition is weaker: we need that the probability of realizations such that $f_{h}(i)=0$ has measure zero.
} 
Lemma 1 With the atomless signal, the full commitment solution of Proposition 1 and the Cases 1 and 2 of the no-commitment solution (Proposition 2) are unchanged.

Remark 2. The threshold $\nu^{2}$ delimiting Cases 2 and 3 will obviously change.

From now on, we concentrate on Case 3 of Proposition 2. When the low cost agent accepts the first-period offer with probability $\gamma$, the second-period principal's beliefs after signal $i$ are $\nu_{2}^{i}=f_{i}(l)$. In the second period the principal chooses between making a high offer $t_{2}=\bar{\theta}$ and obtaining payoff $S-\bar{\theta}$ or targeting the low cost type proposing $t_{2}=\underline{\theta}$ and obtaining payoff $\nu_{2}^{i}(S-\underline{\theta})$. She chooses the first option is $\nu_{2}^{i}$ is lower than $\nu^{1}$ defined in (1) and the second option if it is higher. The low cost type always gets some rent if he is in the second period.

The equilibrium is then the following. The principal makes the first-period offer of $t_{1}=\underline{\theta}+\underline{U}_{1}<\bar{\theta}$, where $\underline{U}_{1}$ is the rent of the low cost type (to be determined below). This offer is accepted by the low cost type with probability $\gamma$ and rejected (with probability 1 ) by the high cost type. In the second period, after having observed some realization of the signal, the principal makes an offer $t_{2}=\underline{\theta}$ or $\bar{\theta}$ depending on the updated probability of the low cost type. If the offer is high, $\bar{\theta}$, both types accept it, if it is low, $\underline{\theta}$, only the low cost type does. For the low cost type playing a mixed strategy, his first-period rent must equal the expected second-period rent.

Lemma 2 The payoff of the principal when the low cost type mixes with probability $\gamma$ is

$$
\begin{aligned}
V(\gamma)= & S\left[\nu \gamma+\nu(1-\gamma) \delta+(1-\nu) \delta F_{h}(q(\gamma))\right] \\
& -\nu[\gamma+(1-\gamma) \delta] \underline{\theta}-\nu \delta \Delta \theta F_{l}(q(\gamma))-(1-\nu) \delta \bar{\theta} F_{h}(q(\gamma)),
\end{aligned}
$$

where

$$
q(\gamma) \equiv \frac{S-\bar{\theta}}{\Delta \theta} \frac{1-\nu}{\nu} \frac{1}{1-\gamma}
$$

Proof. The main step is to find the expected rent of the low cost type in the second period. Let $p_{k}$ be the probability that the agent is of type $k$ given that the first-period offer has been accepted by the low cost type with probability $\gamma$

$$
\begin{aligned}
& p_{l}=\frac{\nu(1-\gamma)}{\nu(1-\gamma)+1-\nu} \text { and } \\
& p_{h}=\frac{1-\nu}{\nu(1-\gamma)+1-\nu} .
\end{aligned}
$$

It is easier to work with the hazard rate

$$
\frac{\nu_{2}^{i}}{1-\nu_{2}^{i}}=\frac{f_{i}(l)}{f_{i}(h)}=\frac{p_{l}}{p_{h}} \frac{f_{l}(i)}{f_{h}(i)}=\frac{\nu}{1-\nu}(1-\gamma) \frac{f_{l}(i)}{f_{h}(i)}
$$


The principal makes the high offer in the second period when this hazard rate is lower than $\frac{\nu^{1}}{1-\nu^{1}}=\frac{S-\bar{\theta}}{\Delta \theta}$. This is the case when

$$
\frac{\nu_{2}^{i}}{1-\nu_{2}^{i}} \leq \frac{S-\bar{\theta}}{\Delta \theta} \Leftrightarrow \frac{f_{l}(i)}{f_{h}(i)} \leq \frac{S-\bar{\theta}}{\Delta \theta} \frac{1-\nu}{\nu} \frac{1}{1-\gamma} \Leftrightarrow \frac{f_{l}(i)}{f_{h}(i)} \leq q(\gamma) .
$$

The low cost type gets rent equal to $\Delta \theta$ in such a realization. His expected rent is

$$
\underline{U}_{2}=\delta \Delta \theta \operatorname{Pr}\left\{\frac{\nu_{2}^{i}}{1-\nu_{2}^{i}} \leq \frac{S-\bar{\theta}}{\Delta \theta} \mid \text { low cost }\right\}=\delta \Delta \theta F_{l}(q(\gamma)) .
$$

The high cost type produces in the same realizations, i.e. when the offer is high. The probability of the production by the high cost type in the second period is then

$$
\operatorname{Pr}\left\{\frac{\nu_{2}^{i}}{1-\nu_{2}^{i}} \leq \frac{S-\bar{\theta}}{\Delta \theta} \mid \text { high cost }\right\}=F_{h}(q(\gamma)) .
$$

Then, the payoff of the principal (7) is (i) expected production benefits that occur in the first period with probability $\nu \gamma$ and in the second period with probability $\nu(1-\gamma)$ by the low cost type and with probability $(1-\nu) F_{h}(q(\gamma))$ by the high cost type; (ii) reimbursement of the low cost type for the production costs; (iii) the rent paid to the low cost type, the share $\gamma$ of which is paid in the first period and the rest in the second period; (iv) the production costs of the high cost type in the second period.

Now, with a continuous signal, there are many mixing probabilities of the low cost type that can be a part of a PBE. In particular, there is an equilibrium when $\gamma=1$, i.e., Case 2 when the low cost agent produces only in the first period. Focusing on the one which gives maximum payoff for the principal, we have to maximize the surplus (7) with respect to $\gamma$.

Proposition 8 If $f_{l}^{\prime}(q(\gamma)) \geq-\frac{1+\gamma}{\gamma} f_{h}(q(\gamma))$, the optimal mixing probability of the low type $\gamma^{*}>0$ is given by the first-order condition

$$
\frac{\nu}{1-\nu} \frac{1}{\nu^{1}} \frac{1-\delta}{\delta}=\frac{\gamma^{*}}{\left(1-\gamma^{*}\right)^{2}} f_{l}\left(q\left(\gamma^{*}\right)\right)
$$

where $q(\gamma)$ is (8).

Proof. Differentiate (7) with respect to $\gamma$ to obtain (10). Differentiate once more to obtain the sufficient second-order condition.

The crucial feature to note from (10) is that the optimal mixing probability $\gamma^{*}$ depends only on the probability $f_{l}\left(q\left(\gamma^{*}\right)\right)$ (and $f_{h}\left(q\left(\gamma^{*}\right)\right)$ ). Therefore, any change in the signal that keeps this probability constant does not affect the optimal delay. Indeed, from (7) 
a higher $\gamma$, and so, a higher $q(\gamma)$ increase the surplus from the production as the low cost type produces more often in the first period and the high cost type produces more often in the second period instead of not producing at all. The costs of this increase are higher production costs and a higher rent for the low cost type. At the margin it is the probabilities at the threshold $q\left(\gamma^{*}\right)$ of all these events that matter, i.e., $f_{l}\left(q\left(\gamma^{*}\right)\right)$ and $f_{h}\left(q\left(\gamma^{*}\right)\right)$.

The optimal mixing probability $\gamma^{*}$ may equal one if the limit of the right hand side is finite when $\gamma$ approaches one.

As a comparative statics exercise, consider the effect of an increase in the discount factor $\delta$. The derivative of condition (10) with respect to $\delta$ is negative (putting all the terms at the left-hand side), i.e., a higher discount factor means more delay. ${ }^{10}$

Let us now see what can happen if the distribution of the signal changes. Assume that it depends on some parameter $a$, i.e., it is $f_{l}(i, a)$. A change in $a$ must necessarily increase $f_{l}(i, a)$ for some realizations and decrease for others in order to keep $\int_{0}^{+\infty} f_{l}(i, a) d i$ equal one. Then, depending on the realization $q\left(\gamma^{*}\right)$, any change in the distribution has an ambiguous effect on $\gamma^{*}$.

To conclude the analysis of the "0-1" case, let us comment what happens when the signal has a discreet support with more than one non-perfect realizations (in signal (3) there is only one non-perfect realization). The analysis combines the insights from this section and from the previous one. As in the continuous signal case, when the low cost type mixing probability $\gamma$ changes, so does the realization in which the principal is indifferent between making the high and the low offer, i.e., the realization $i$ such that $\nu_{2}^{i}=\nu^{1}{ }^{11}$ "Inside" this realization, the analysis is the same as for the signal (3): the principal chooses her mixing strategy (to offer a high or a low price) and she optimally chooses to offer the low price with probability one.

\subsection{The general case of the "variable quantity" model}

Now we come back to the variable quantity model. We take the setup of Section 2.2 and we add a signal in the sense specified in Section 3 that arrives between the two periods. Even when the signal is simple as the one considered in Section 3.2, the analysis is quite involved and can be easily generalized. Thus, we will not impose any restrictions on the signal here. In the next Section we come back to the simple signal (3).

\footnotetext{
${ }^{10}$ The sign of $V_{\gamma \delta}^{\prime \prime}$ is the same as the sign of $\gamma_{\delta}^{\prime}$ since differentiating $V_{\gamma}^{\prime}=0$ yields $\gamma_{\delta}^{\prime}=-\frac{V_{\gamma \delta}^{\prime \prime}}{V_{\gamma \gamma}^{\prime \prime}}$.

${ }^{11}$ It could be that $\gamma$ is such that in any realization the principal strictly prefers to make one or the other offer (i.e., the realization in which she is indifferent does not exist; it is "between" two adjacent realizations $i$ and $i+1)$. However, it is not optimal: the principal gains and the agent is indifferent if $\gamma$ increases up to a value such that the principal is indifferent in realization $i+1$.
} 
Under no commitment, the principal proposes a first-period menu $\left\{\left(\underline{q}_{1}, \underline{t}_{1}\right),\left(\bar{q}_{1}, \bar{t}_{1}\right)\right\}$ designed for the low and high cost agent, respectively. ${ }^{12}$ If this contract is rejected, a signal $i$ realizes and the principal proposes a second-period menu $\left\{\left(\underline{q}_{2}^{i}, \underline{t}_{2}^{i}\right),\left(\bar{q}_{2}^{i}, \bar{t}_{2}^{i}\right)\right\}$ that the agent accepts with probability one as Section 3.4.1 shows. As noted earlier, we look for an optimal $\mathrm{PBE}$ of the game, i.e. the one that gives the maximum payoff to the principal.

Denote by $\underline{\alpha}$ the acceptance probability of the first-period contract by the low cost type and by $\bar{\alpha}$ that of the high cost type. ${ }^{13}$ These acceptance probabilities can be interpreted as indicating the delay in production: a higher acceptance probability of a type means less delay (less waiting) for this type. If $\underline{\alpha}=\bar{\alpha}=1$ there is no delay, and if $\underline{\alpha}=\bar{\alpha}=0$ there is maximum (full) delay. These probabilities determine (in expectation) the contracting in the second period. It turns out to be extremely useful to characterize a PBE by the firstperiod probabilities $(\underline{\alpha}, \bar{\alpha})$. As Lemmas 4,5 and 6 below show for any pair $(\underline{\alpha}, \bar{\alpha})$ there is in general either a unique or no first-period offer that is consistent with this pair. ${ }^{14}$ In what follows we call pure strategy $\mathrm{PBE}$ an equilibrium where the first-period probabilities $(\underline{\alpha}, \bar{\alpha})$ are either zero or one. Otherwise it is a mixed strategy PBE.

We will proceed assuming a continuous support of the signal distribution with the normalization $\frac{f_{l}(i)}{f_{h}(i)} \equiv i$ introduced in Section 3.3. Unlike the "0-1" model, the analysis and the results do not change whether the support of the signal is continuous or discrete; it matters only for notation. The only difference comes if $\sum_{i: f_{h}(i)>0} f_{l}(i)<1$ (i.e., the realizations such that $f_{h}(i)=0$ have a probability of a positive measure). We discuss when it is relevant what happens when $\sum_{i: f_{h}(i)>0} f_{l}(i)$ is less than one or equal to one.

\footnotetext{
${ }^{12}$ This is a dynamic game with no commitment, thus, we cannot use the Revelation Principle. Any assumption on the contractual space should be justified.

We restrict the principal to propose deterministic contracts. Then, there is no loss of generality in considering message spaces with three messages (i.e. first-period menu consisting of two contracts and no contract or two contracts with the possibility that the agent refuses as here) in the following sense. For any bigger message space and for any incentive efficient equilibrium in the terminology of Bester and Strausz (2001) (i.e. an equilibrium in which the principal receives the maximal payoff under the condition that the agent gets a certain rent) there is a payoff-equivalent equilibrium when the message space is of cardinality three. Moreover, if the agent chooses to produce in the first period, he reveals his type truthfully. As there is no continuation in this case, the agent does not have an incentive to mispresent his type.

I am currently working on the general case when the principal can propose stochastic contracts. Applying the result of Bester and Strausz (2001) we can consider menus of only two contracts as there are two types. Due to the agent's risk-neutrality each contract is a lottery with at most two elements: some first-period production/transfer and no contract. Preliminary results show that under some conditions equilibria identified in the present paper still remain equilibria in the general case.

${ }^{13}$ In the second period in equilibrium agents of either type always accept the contract.

${ }^{14}$ If one (or both) acceptance probabilities is zero there are many offers consistent with such probabilities. These cases are of no interest since the agent never picks these offers.
} 


\subsubsection{The second period}

Since the principal makes the second-period offer after the signal is realized contracting is different after each realization. In the next subsection we derive the optimal secondperiod contract after a given realization. Then, we compute the expected second-period payoff of the principal.

Contracting in the second period after a given realization of the signal After the first-period offer was rejected and the principal updated her beliefs after a signal $i$, she solves the standard Baron-Myerson adverse selection problem. She proposes a secondperiod menu $\left\{\left(\underline{q}_{2}^{i}, \underline{t}_{2}^{i}\right),\left(\bar{q}_{2}^{i}, \bar{t}_{2}^{i}\right)\right\}$ maximizing her payoff subject to a participation constraint $(P)$ and an incentive compatibility constraint $(I C)$ for each type:

$$
\left\{\begin{array}{cl}
\max _{\underline{q}_{2}^{i}, \underline{t}_{2}^{i}, \bar{q}_{2}^{i}, \bar{t}_{2}^{i}} & f_{i}(l)\left(S\left(\underline{q}_{2}^{i}\right)-\underline{t}_{2}^{i}\right)+f_{i}(h)\left(S\left(\bar{q}_{2}^{i}\right)-\bar{t}_{2}^{i}\right) \\
\text { s.t. } & \bar{t}_{2}^{i}-\bar{\theta} \bar{q}_{2}^{i} \geq 0, \\
\bar{P} & \underline{t}_{2}^{i}-\underline{\theta} \underline{q}_{2}^{i} \geq 0, \\
\underline{I C} & \bar{t}_{2}^{i}-\bar{\theta} \bar{q}_{2}^{i} \geq \underline{t}_{2}^{i}-\bar{\theta} \underline{q}_{2}^{i}, \\
\underline{I C} & \underline{t}_{2}^{i}-\underline{\theta} \underline{q}_{2}^{i} \geq \bar{t}_{2}^{i}-\underline{q}_{2}^{i} .
\end{array}\right.
$$

Replace transfers by quantities $\bar{U}_{2}^{i} \equiv \bar{t}_{2}^{i}-\bar{\theta} \bar{q}_{2}^{i}$ and $\underline{U}_{2}^{i} \equiv \underline{t}_{2}^{i}-\underline{\theta}_{2}^{i}$ and obtain the following Lemma.

Lemma 3 After a realization $i$, the optimal offer of the principal $\left\{\left(\underline{q}_{2}^{i}, \underline{U}_{2}^{i}\right),\left(\bar{q}_{2}^{i}, \bar{U}_{2}^{i}\right)\right\}$ is

$$
\begin{aligned}
& \underline{q}_{2}^{i}=\underline{q}^{F B}, \text { solution of } S^{\prime}\left(\underline{q}^{F B}\right)=\underline{\theta}, \\
& \bar{q}_{2}^{i}, \text { solution of } S^{\prime}\left(\bar{q}_{2}^{i}\right)=\bar{\theta}+\frac{f_{i}(l)}{f_{i}(h)} \Delta \theta, \\
& \underline{U}_{2}^{i}=\Delta \theta \bar{q}_{2}^{i}, \\
& \bar{U}_{2}^{i}=0 .
\end{aligned}
$$

The expression for the high cost type quantity can be written as

$$
S^{\prime}\left(\bar{q}_{2}^{i}\right)=\bar{\theta}+\frac{\nu}{1-\nu} \frac{1-\underline{\alpha}}{1-\bar{\alpha}} \frac{f_{l}(i)}{f_{h}(i)} \Delta \theta
$$

Proof. As usual, two constraints bind: the participation constraint of the high cost agent $(\bar{P})$ and the incentive compatibility of the low cost agent $(\underline{I C})$. We obtain the standard Baron-Myerson contract (12).

We have

$$
\begin{aligned}
& p_{l}=\frac{\nu(1-\underline{\alpha})}{\nu(1-\underline{\alpha})+(1-\nu)(1-\bar{\alpha})} \text { and } \\
& p_{h}=\frac{(1-\nu)(1-\bar{\alpha})}{\nu(1-\underline{\alpha})+(1-\nu)(1-\bar{\alpha})} .
\end{aligned}
$$


Then,

$$
\frac{f_{i}(l)}{f_{i}(h)}=\frac{p_{i l}}{p_{i h}}=\frac{p_{l}}{p_{h}} \frac{f_{l}(i)}{f_{h}(i)}=\frac{\nu}{1-\nu} \frac{1-\underline{\alpha}}{1-\bar{\alpha}} \frac{f_{l}(i)}{f_{h}(i)} .
$$

With this expression of the revision of beliefs, we obtain (13).

The expected second-period payoff of the principal is

$$
V_{2}^{i}=f_{i}(l)\left[S\left(\underline{q}^{F B}\right)-\underline{\theta}^{F B}\right]-f_{i}(l) \Delta \theta \bar{q}_{2}^{i}+f_{i}(h)\left[S\left(\bar{q}_{2}^{i}\right)-\bar{\theta} \bar{q}_{2}^{i}\right] .
$$

In the rest of this subsection we discuss (13). The denominator of the second ratio in the right hand side is zero if $\bar{\alpha}=1$ and/or if $f_{h}(i)=0$. In words, the revised probability that the agent is high cost is zero if the high cost agent always accepts the first-period contract and/or the information indicates that after this particular signal $i$ the agent is surely low cost. In this case the principal proposes the null contract to the high cost type, i.e., $\bar{q}_{2}^{i}=0$, that leaves no rent to the low cost agent: $\underline{U}_{2}^{i}=\Delta \theta \bar{q}_{2}^{i}=0$.

When both the numerator and denominator of the right hand side of (13) are zero the principal cannot update her beliefs. When the support of the signal is atomless, this is only possible (with a probability of positive measure) when $\underline{\alpha}=\bar{\alpha}=1$. When the support is discrete, there can be other cases as well. For example, when the low cost type always accepts the first-period offer, i.e., $\underline{\alpha}=1$, and the signal $i$ cannot be realized if the type is high cost: $f_{h}(i)=0$.

This is impossible on the equilibrium path. However, payoffs in these off-equilibrium situations define outside options on the equilibrium path. The rent that the principal proposes in these cases puts a lower bound on the rent that the agent has on the equilibrium path. Therefore, the "best" out-of-equilibrium beliefs give to the agent the least incentives to deviate. We make the following assumption.

Assumption 1 In off-the-equilibrium-path events (i.e., when $\frac{f_{i}(l)}{f_{i}(h)}$ is not defined) the principal believes that the agent is low cost. She proposes the following contract:

- The low cost agent produces at the first-best level, $\underline{q}_{2}^{i}=\underline{q}^{F B}$, and receives no rent, $\underline{U}_{2}^{i}=0$.

- The high cost agent does not produce, $\bar{q}_{2}^{i}=0$, and receives no rent, $\bar{U}_{2}^{i}=0$.

These beliefs are "reasonable" if there exist arbitrarily small positive costs of going to the second period borne by the agent, as we suggested earlier in Remark after the proof of Proposition 4. The principal might propose the reimbursement of these costs when she wants. Then, the high cost type never wants to reject a first-period offer that gives him zero rent. Any off-equilibrium situation will be caused by a deviation of the low cost type.

Assumption 1 makes the principal's profit function continuous with respect to $\underline{\alpha}$ which simplifies the analysis. See details in Appendix A. 
Expected payoffs of the second period In this subsection we derive the payoffs of the principal and her agent when the agent refused the first-period offer and before the signal realizes. These expected second-period payoffs affect the first-period contract studied later in the next Section.

Let $x^{i}$ be a random variable over realizations of the signal. Then $E\left[x^{i}\right]$ is the unconditional expectation and $E_{k}\left[x^{i}\right]$ is the conditional expectation of $x^{i}$ given that the agent is of type $k$ :

$$
\begin{aligned}
& E\left[x^{i}\right] \equiv \int_{0}^{+\infty} x^{i} f(i) d i, \\
& E_{k}\left[x^{i}\right] \equiv \int_{0}^{+\infty} x^{i} f_{k}(i) d i .
\end{aligned}
$$

Denote by $W(q)$ the social surplus from the production

$$
\begin{aligned}
& \underline{W}(q) \equiv S(q)-\underline{\theta} q, \\
& \bar{W}(q) \equiv S(q)-\bar{\theta} q .
\end{aligned}
$$

For example, in the second period after a signal $i$ the social surplus from the production of the high cost type is $\bar{W}\left(\bar{q}_{2}^{i}\right)$. The expected social surplus from his production in the second period is $E_{h}\left[\bar{W}\left(\bar{q}_{2}^{i}\right)\right]$.

If the first-period offer is refused, the expected second-period profit of the principal is $\left(V_{2}^{i}\right.$ is given by $\left.(15)\right)$

$$
V_{2} \equiv E\left[V_{2}^{i}\right]=p_{l} \underline{W}\left(\underline{q}^{F B}\right)-p_{l} \Delta \theta E_{l}\left[\bar{q}_{2}^{i}\right]+p_{h} E_{h}\left[\bar{W}\left(\bar{q}_{2}^{i}\right)\right] .
$$

The first term of this expression is the value of production by the low cost type; it happens with probability $p_{l}$. The second term is expected rent paid to the low cost agent; since it is paid only when the agent is low cost, its expectation should be conditioned on the agent being low cost. The last term is the value of production by the high cost agent; it should be conditioned on the cost being high.

\subsubsection{The first period}

Optimal first-period offer given acceptance probabilities $(\underline{\alpha}, \bar{\alpha}) \quad$ Now we analyze the first period. In this Section, we characterize the first-period offer $\left\{\left(\underline{q}_{1}, \underline{t}_{1}\right),\left(\bar{q}_{1}, \bar{t}_{1}\right)\right\}$ given that the agent plays some acceptance probabilities $(\underline{\alpha}, \bar{\alpha})$. In other words, for any pair $(\underline{\alpha}, \bar{\alpha})$ we look for a first-period offer such that this offer and the pair $(\underline{\alpha}, \bar{\alpha})$ form some PBE.

It is useful to introduce the notation

$$
\lambda=\frac{1-\underline{\alpha}}{1-\bar{\alpha}}
$$


Clearly, for any $\lambda$ there is a continuum of pairs $(\underline{\alpha}, \bar{\alpha})$ that correspond to this $\lambda$. We can write the second-period quantity (13) as a function of $\lambda$ :

$$
S^{\prime}\left(\bar{q}_{2}^{i}(\lambda)\right)=\bar{\theta}+\frac{\nu}{1-\nu} \lambda \frac{f_{l}(i)}{f_{h}(i)} \Delta \theta
$$

If $\lambda=0(\underline{\alpha}=1)$, then the principal is sure to meet the high cost type agent in the second period, so $\bar{q}_{2}^{i}=q_{2}^{F B}$ for all $i$. When $\lambda=+\infty(\bar{\alpha}=1)$ the principal is sure to meet the low cost agent, and $\bar{q}_{2}^{i}=0$ for all $i$. Because $S(q)$ is concave, $\bar{q}_{2}^{i}$ decreasing in $\lambda$ (and increasing in $\underline{\alpha}$ ). The intuition is that a higher $\lambda$ or a lower $\underline{\alpha}$ mean a smaller probability of the low cost type, so the rent-efficiency trade-off changes in favor of a less distortion for the high cost type.

Now we find the optimal first-period offer given that the agent's acceptance probabilities result in $\lambda$; we say that this first-period offer induces $\lambda$. We do it separately for $\underline{\alpha}<1$ and $\underline{\alpha}=1$. In Lemmas 4 and 5 we proceed taking both $\underline{\alpha}$ and $\bar{\alpha}$ positive so that relevant constraints must be satisfied for each type. In Lemma 6 we prove that results are not altered when one of them is zero.

As usual, we can replace transfers by rents in the principal's problem: $\bar{U}_{1} \equiv \bar{t}_{1}-\bar{\theta} \bar{q}_{1}$ and $\underline{U}_{1} \equiv \underline{t}_{1}-\underline{\theta}_{1}$ and write the first-period offer in the form $\left\{\left(\underline{q}_{1}, \underline{U}_{1}\right),\left(\bar{q}_{1}, \bar{U}_{1}\right)\right\}$. Lemma 4 characterizes this menu for $\underline{\alpha}<1$. Its main result is that the quantity for the high cost type is equal to his discounted expected second-period quantity: $\bar{q}_{1}=\delta E_{l}\left[\bar{q}_{2}^{i}(\lambda)\right]$. Indeed, the low cost agent must receive the same rent in the first period as in the second one. In the first period he receives $\Delta \theta \bar{q}_{1}$ and the expected discounted rent of the second period is $\delta \Delta \theta E_{l}\left[\bar{q}_{2}(\lambda)\right]$. Therefore, the menu is similar to the standard second-best menu, only the downward distortion for the high cost type is determined by intertemporal considerations and not by the standard rent-efficiency trade-off.

Lemma 4 For $0<\underline{\alpha}<1$ and $\bar{\alpha}>0$ the first-period offer $\left\{\left(\underline{q}_{1}, \underline{U}_{1}\right),\left(\bar{q}_{1}, \bar{U}_{1}\right)\right\}$ is characterized by:

- The low cost agent produces at the first-best level, $\underline{q}_{1}=\underline{q}^{F B}$, and earns a positive rent, $\underline{U}_{1}=\Delta \theta \bar{q}_{1}$.

- The high cost agent's quantity is equal to his discounted expected second-period quantity, $\bar{q}_{1}=\delta E_{l}\left[\bar{q}_{2}^{i}(\lambda)\right]$, and his rent is zero, $\bar{U}_{1}=0$.

Proof. For given $(\underline{\alpha}, \bar{\alpha})$ the second period payoffs are completely determined. The principal maximizes her payoff in the first period which is a standard second-best problem with two new constraints, $\underline{I C}_{i n t}$ and $\overline{I C}_{i n t}$ for the low and high cost type, respectively. ${ }^{15}$

\footnotetext{
${ }^{15}$ If $\bar{\alpha}=1$, the high cost type does not play a mixed strategy, so his $\overline{I C}_{i n t}$ constraint becomes inequality. This does not matter as this type always receives zero rent.
} 
These are intertemporal incentive compatibility constraints: as both types play a mixed strategy they have to receive the same utility in the two periods.

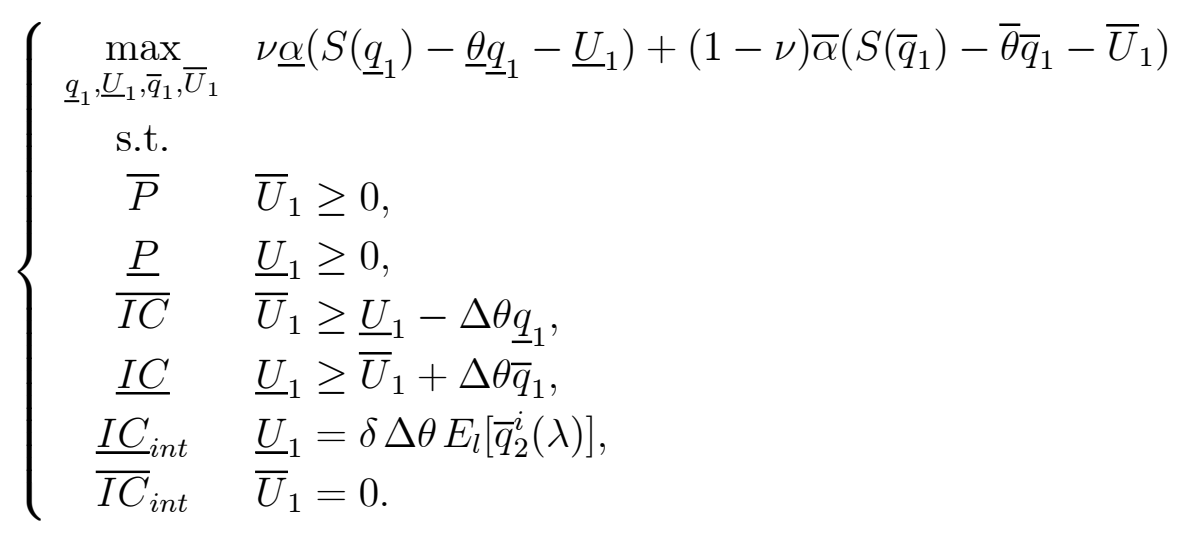

To see that the $\underline{I C}$ constraint binds note that $\delta E_{l}\left[\bar{q}_{2}^{i}(\lambda)\right]<\bar{q}^{F B}$. If the $\underline{I C}$ constraint does not bind, the principal wants to increase the first-period high cost quantity $\bar{q}_{1}$ as it increases her surplus without violating any constraint. The principal will increase it until the $\underline{I C}$ constraint binds, i.e., when $\bar{q}_{1}=\delta E_{l}\left[\bar{q}_{2}^{i}(\lambda)\right]$.

The low cost type's production is not distorted as it does not enter in expressions for the rents.

Let us move to the case when the low cost type always accepts the first-period offer $(\underline{\alpha}=1)$. Then, in the second period the principal faces only the high cost type and, therefore, contracts at the first-best level. The $\underline{I C}_{\text {int }}$ constraint becomes ${ }^{16}$

$$
\underline{I C}_{i n t}: \underline{U}_{1} \geq \delta \Delta \theta \bar{q}^{F B}
$$

If (20) binds, the first-period offer is the same as in the case $\underline{\alpha}<1$ : the production of the high cost type is given by his discounted expected second-period production. Since $\underline{\alpha}=1$ the principal will propose the first-best contract for the high cost type in the secondperiod along the equilibrium path. ${ }^{17}$ Then, $\bar{q}_{1}=\delta E_{l}\left[\bar{q}_{2}^{i}(\lambda)\right]=\delta \bar{q}^{F B}$. The first-period offer is the same as the one identified in Lemma 4.

If the constraint (20) does not bind, in the first period the principal solves a standard adverse-selection problem with probability $\nu$ of the low cost type and probability $(1-\nu) \bar{\alpha}$

\footnotetext{
${ }^{16}$ For a discrete support this constraint is

$$
\underline{I C}_{i n t}: \underline{U}_{1} \geq \delta \Delta \theta \bar{q}^{F B} \sum_{i: f_{h}(i)>0} f_{l}(i)
$$

for Assumption 1 sets $\bar{q}_{2}^{i}$ equal to zero when $f_{h}(i)$ is zero.

${ }^{17}$ If the low cost type deviates, his deviation comes unnoticed unless the realization is such that $f_{h}(i)=$ 0 . In this case, the principal cannot revise her beliefs as (14) shows. By Assumption 1, in these cases the principal does not leave any rent to the low cost type. With atomless distribution, the probability of these realizations has measure zero.
} 
of the high cost type. Note that if the high cost type always accepts in the first period (i.e., $\bar{\alpha}=1$ ) then the principal will not give any rent in the second period, so the constraint (20) is not relevant in this case. This is summarized in Lemma 5.

Lemma 5 For $\underline{\alpha}=1$ and $\bar{\alpha}>0$ the first-period contract $\left\{\left(\underline{q}_{1}, \underline{U}_{1}\right),\left(\bar{q}_{1}, \bar{U}_{1}\right)\right\}$ is characterized by:

- The low cost agent produces at the first-best level, $\underline{q}_{1}=\underline{q}^{F B}$, and earns a positive rent, $\underline{U}_{1}=\Delta \theta \bar{q}_{1}$.

- The high cost agent earns no rent, $\bar{U}_{1}=0$, and his production is equal to

- if $\bar{\alpha}=1: \bar{q}_{1}=\bar{q}_{1}(\bar{\alpha})$ solution of

$$
S^{\prime}\left(\bar{q}_{1}(\bar{\alpha})\right)=\bar{\theta}+\frac{\nu}{1-\nu} \frac{1}{\bar{\alpha}} \Delta \theta
$$

- if $\bar{\alpha}<1$ : the maximum of his discounted expected second-period quantity and the second-best quantity with probabilities $\nu$ and $(1-\nu) \bar{\alpha}$ of the low cost and the high cost type, respectively: $\bar{q}_{1}=\max \left\{\delta \bar{q}^{F B}, \bar{q}_{1}(\bar{\alpha})\right\}$.

Proof. See Appendix B.

In Lemmas 4 and 5 we assumed that both types produce in the first period with some probability. If some type does not produce, then its constraints change: the individual rationality constraint disappears, and the intertemporal incentive compatibility constraint becomes an inequality. The next Lemma shows that menus found in Lemmas 4 and 5 remain optimal.

Lemma 6 Lemma 4 holds when either $\underline{\alpha}$ or $\bar{\alpha}$ (or both) is equal to zero.

Lemma 5 holds when $\bar{\alpha}$ is equal to zero.

Proof. See Appendix B.

Thanks to the three Lemmas of this section we can write the payoff of the principal for a given pair $(\underline{\alpha}, \bar{\alpha})$ and study her preferences over different pairs. In the next section, I show that it is not optimal for the principal to induce mixed strategies for both types, i.e., if both $\underline{\alpha}$ and $\bar{\alpha}$ are strictly between 0 and 1 her profits are not maximized (Proposition 9 ). Then at least one type plays a pure strategy which is studied in Section afterwards. 
A proof that at least one type plays a pure strategy In this section we consider the case when both types play a mixed strategy, i.e., both $\underline{\alpha}$ and $\bar{\alpha}$ are strictly between 0 and 1 . We prove that this cannot be optimal for the principal.

Let us write the principal's intertemporal profit function. Lemma 4 establishes that if the low cost type plays a mixed strategy, then $\bar{q}_{1}=\delta E_{l}\left[\bar{q}_{2}^{i}\right]$. His expected rent $\underline{U}$ is then $\Delta \theta \bar{q}_{1}$ and it is paid partly in the first period and partly in the second one. The principal's intertemporal profits are then

$$
\begin{aligned}
& V(\underline{\alpha}, \bar{\alpha})=\nu \underline{W}\left(\underline{q}^{F B}\right)(\underline{\alpha}+\delta(1-\underline{\alpha})) \quad V_{1} \\
& -\nu \Delta \theta \bar{q}_{1} \quad V_{2} \\
& +(1-\nu) \bar{\alpha} \bar{W}\left(\bar{q}_{1}\right) \quad V_{3} \\
& +(1-\nu)(1-\bar{\alpha}) \delta E_{h}\left[\bar{W}\left(\bar{q}_{2}^{i}\right)\right] \quad V_{4}
\end{aligned}
$$

The quantities of the high cost type $\bar{q}_{1}$ and $\bar{q}_{2}^{i}$ depend on $\underline{\alpha}$ and $\bar{\alpha}$; for notational simplicity we omitted the arguments.

The first term $V_{1}$ is the surplus from the low cost type: the production is always at the first-best level and with probability $1-\underline{\alpha}$ it happens in the second period. $V_{2}$ is the expected rent to the low cost type. $V_{3}$ is the first-period surplus from the high cost agent. $V_{4}$ is the expected second-period surplus from the high cost type.

Now we are ready to prove the announced result of this section.

Proposition 9 In an optimal equilibrium at least one type plays a pure strategy.

Proof. See Appendix B.

The logic of the proof is the following. We want to prove that when both types play a mixed strategy, the principal's profits are not maximized, i.e., the first-order and secondorder conditions for the maximization of $V(\underline{\alpha}, \bar{\alpha})$ cannot be satisfied simultaneously. Make a change of variables in $V(\underline{\alpha}, \bar{\alpha})$ : replace $\underline{\alpha}$ for $\lambda=\frac{1-\underline{\alpha}}{1-\bar{\alpha}}$. This gives a new profit function $\widetilde{V}(\lambda, \bar{\alpha})$. It is linear with respect to $\bar{\alpha}$ since $\bar{q}_{2}^{i}$ depends now only on $\lambda$. The second-order conditions are $\widetilde{V}_{\lambda \lambda}^{\prime \prime} \leq 0$ and $\widetilde{V}_{\lambda \bar{\alpha}}^{\prime \prime}=0$. The three conditions $\widetilde{V}_{\bar{\alpha}}^{\prime}=\widetilde{V}_{\lambda}^{\prime}=\widetilde{V}_{\lambda \bar{\alpha}}^{\prime \prime}=0$ turn out to be incompatible.

Optimal in-pair equilibria Proposition 9 shows that at least one type plays a pure strategy. Therefore, there are four possible cases of acceptance probabilities $\underline{\alpha}$ and $\bar{\alpha}$ : $(\underline{\alpha}, 1),(\underline{\alpha}, 0),(1, \bar{\alpha})$ and $(0, \bar{\alpha})$. Proposition 10 establishes that the principal's profits reach the maximum at one of the three points: $(1,1),(1,0)$ or $(\underline{\alpha}, 0)$ with $\underline{\alpha}>0$.

These results can be interpreted as follows. First, in any equilibrium with delay only the low cost type produces in the first period. If the first-period offer is refused, the principal becomes more pessimistic about the agent's type. This result implies that the 
high cost agent never plays a mixed strategy — in any equilibrium, he always accepts the first-period offer with probability either 0 or 1.

Second, in any equilibrium there is production in the first period with a strictly positive probability. In an equilibrium with delay the low cost type produces with a strictly positive probability. This holds even if the future information is perfect, i.e., such that will reveal completely the agent's type. The intuition is that if both principal and agent know that in the second period there will be no asymmetric information and thus zero rent for the agent, the principal will propose and the agent will accept a first-period contract with a small rent.

Proposition 10 The principal's profits are maximal when the agent's strategy $(\underline{\alpha}, \bar{\alpha})$ is one of the following three strategies:

- No delay $(1,1)$;

- Delay for the high cost type $(1,0)$;

- Delay for the high cost type and partial delay for the low cost type $(\underline{\alpha}, 0)$, where $\underline{\alpha}>0$ is given by (if it exists)

$$
V_{\underline{\alpha}}^{\prime}(\underline{\alpha}, 0)=\nu\left[(1-\delta) \underline{W}\left(\underline{q}^{F B}\right)-\delta \underline{\alpha} \Delta \theta E_{l}\left[\bar{q}_{2 \underline{\alpha}}^{\prime \prime}\right]\right]=0 .
$$

Proof. See Appendix B.

Remark 3. If probability of realizations such that $f_{h}(i)=0$ has measure zero (for example, atomless distribution), then no delay $(1,1)$ dominates delay for the high cost type $(1,0) \cdot{ }^{18}$

Remark 4. A sufficient second-order condition for (23) to give the global maximum for $(\underline{\alpha}, 0)$ solution is $S^{\prime \prime \prime}(q) \geq 0$.

In order to work with (23) we assume through the rest of the paper that it does give the optimal partial delay, i.e., that $S^{\prime \prime \prime}(q)$ is positive. ${ }^{19}$

\footnotetext{
${ }^{18}$ In this case the principal has to pay rent equal to $\delta \Delta \theta \bar{q}^{F B}$ to the low cost type in the first period to prevent his deviation. Then,

$$
\begin{aligned}
V(1,0) & =\nu \underline{W}\left(\underline{q}^{F B}\right)-\nu \Delta \theta \delta \bar{q}^{F B}+(1-\nu) \delta \bar{W}\left(\bar{q}^{F B}\right) \\
& <\nu \underline{W}\left(\underline{q}^{F B}\right)-\nu \Delta \theta \delta \bar{q}^{F B}+(1-\nu) \bar{W}\left(\delta \bar{q}^{F B}\right) \\
& \leq \nu \underline{W}\left(\underline{q}^{F B}\right)-\nu \Delta \theta \bar{q}^{S B}+(1-\nu) \bar{W}\left(\bar{q}^{S B}\right)=V(1,1) .
\end{aligned}
$$

The first inequality follows from the concavity of $\bar{W}(q)$; the second one follows from the definition of $\bar{q}^{S B}$ (equality is reached in a non-generic case when $\delta \bar{q}^{F B}=\bar{q}^{S B}$ ).

${ }^{19}$ The case of $S^{\prime \prime \prime}(q)=0$ is of no interest as in this case $S(q)$ is quadratic. Then, it cannot satisfy the Inada conditions (it cannot have $S^{\prime}(0)=\infty$ ).
} 
Assumption $2 S^{\prime \prime \prime}(q)$ is positive.

Let us see the difference between the first-order condition (23) for the "variable quantity" model and the first-order condition (10) for the "0-1" model. Here, as the high cost type quantity varies continuously with the principal's beliefs and so with the realization of the signal, any change in the distribution of the signal affect the low cost type expected rent and thus, delay. In the "0-1" model only the probabilities at the threshold $q(\gamma)$ matter.

We can note a more subtle feature of (23) which turns out to be extremely important in applications. Any change in the distribution of the signal might have an ambiguous effect on delay. For example, let $f_{l}(i)$ vary for one particular realization $i .^{20}$ It will only change $f_{l}(i) \bar{q}_{2 \underline{\alpha}}^{i \prime}$ in $(23)$ as follows:

$$
\frac{\partial}{\partial f_{l}(i)}\left[f_{l}(i) \bar{q}_{2 \underline{\alpha}}^{i \prime}\right]=\bar{q}_{2 \underline{\alpha}}^{i \prime}\left(2+(1-\underline{\alpha}) \frac{S^{\prime \prime \prime}\left(\bar{q}_{2}^{i}\right)}{S^{\prime \prime}\left(\bar{q}_{2}^{i}\right)} \bar{q}_{2 \underline{\alpha}}^{i \prime}\right) .
$$

We know the signs of all the elements in (24): $\bar{q}_{2 \underline{\alpha}}^{i \prime}>0$ (a higher $\underline{\alpha}$ makes the principal more pessimistic in the second period and she increases the high cost agent's quantity), $S^{\prime \prime}(q)<0$ (concavity of the principal's surplus function) and $S^{\prime \prime \prime}(q)>0$ (Assumption 2). Then, the term in brackets in (24) may be either positive or negative, i.e., an increase in $f_{l}(i)$ may either increase or decrease $\underline{\alpha}$. Note that a variable quantity alone is not enough to obtain this non-monotonicity, the third derivative of the principal's surplus function must be strictly positive.

Section 3.5 studies the optimal delay for some particular signal (the one with perfect realizations of Section 3.2). It characterizes comparative statics with respect to various parameters.

Comparative statics with respect to the discount factor As a comparative statics exercise let us see how the delay depends on the discount factor. As intuition would suggest, the relation is indeed positive.

We check it in two steps. First, when the principal chooses between no delay $(1,1)$ and delay for the high cost type $(\underline{\alpha}, 0)$ a higher $\delta$ favors the choice of the latter one since its payoff increases with the discount factor while no-delay profits $V(1,1)$ do not depend on it. Second, consider the choice of optimal $\underline{\alpha}$. If the solution to (23) exists we can prove that it is decreasing in the discount factor.

\footnotetext{
${ }^{20}$ There must be another realization $j$ for which $f_{l}(j)$ decreases to keep $\int_{0}^{+\infty} f_{l}(i) d i=1$. Given the non-linearity of the effect of each $f_{l}(i)$ the total effect will still be ambiguous and we can consider a change for only one $i$.
} 
We do it in the following way that we use several times more in Section 3.5. If we take the full derivative of the condition $(23) V_{\underline{\alpha}}^{\prime}=0$ with respect to some parameter (here it is the discount factor) we obtain

$$
\underline{\alpha}_{\delta}^{\prime}=-\frac{V_{\underline{\alpha} \delta}^{\prime \prime}}{V_{\underline{\alpha \alpha}}^{\prime \prime}} .
$$

The denominator is negative, therefore,

$$
\operatorname{sign}\left(\underline{\alpha}_{\delta}^{\prime}\right)=\operatorname{sign}\left(V_{\underline{\alpha} \delta}^{\prime \prime}\right) .
$$

To find how the optimal $\underline{\alpha}$ changes with some parameter, we have to find the sign of the derivative of $V_{\underline{\alpha}}^{\prime}$ with respect to this parameter.

Differentiate $V_{\underline{\alpha}}^{\prime}(\underline{\alpha}, 0)$ in $(23)$ with respect to $\delta$ :

$$
V_{\underline{\alpha} \delta}^{\prime \prime}(\underline{\alpha}, 0)=-\underline{W}\left(\underline{q}^{F B}\right)-\underline{\alpha} \Delta \theta E_{l}\left[\bar{q}_{2 \underline{\alpha}}^{i \prime}\right]<0 .
$$

When $\delta$ decreases, $\underline{\alpha}$ increases which means less waiting. Moreover, for $\delta$ small enough $V_{\underline{\alpha}}^{\prime}(\underline{\alpha}, 0)$ given by $(23)$ is always positive, so $\underline{\alpha}=1$. We established therefore that the optimal (for the principal) delay increases (non-strictly) with the discount factor.

\subsection{Example of a signal in the "variable quantity model"}

Let us consider the same signal as in Section 3.2: with probability $\underline{p}$ there is a perfect realization of the signal if the agent is low cost and $\bar{p}$ if he is high cost. The signal can be represented as (3)

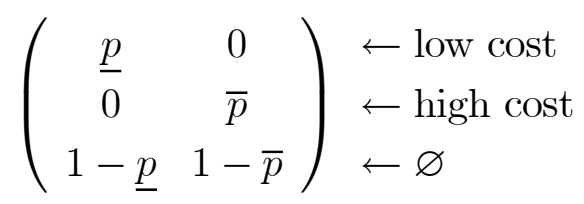

Proposition 10 applies. We focus on equilibria with delay $(\underline{\alpha}, 0)$.

\subsubsection{Optimal delay for a general surplus function}

In the case of rejection of the first-period offer and no perfect realization, in the second period the high cost agent's quantity $\bar{q}_{2}^{n}$ is given by (see (13) for the general case)

$$
S^{\prime}\left(\bar{q}_{2}^{n}\right)=\bar{\theta}+\frac{\nu}{1-\nu}(1-\underline{\alpha}) \frac{1-\underline{p}}{1-\bar{p}} \Delta \theta
$$

The low cost type that refuses the first-period contract gets zero rent (when the principal discovers his type) with probability $\underline{p}$ and he gets $\delta \Delta \theta \bar{q}_{2}^{n}$ with probability $1-\underline{p}$. Therefore, $E_{l}\left[\bar{q}_{2}^{i}\right]=(1-\underline{p}) \bar{q}_{2}^{n}$ and 


$$
E_{l}\left[\bar{q}_{2 \underline{\alpha}}^{i \prime}\right]=(1-\underline{p}) \bar{q}_{2 \underline{\alpha}}^{n \prime}=-\frac{1}{S^{\prime \prime}\left(\bar{q}_{2}^{n}\right)} \frac{\nu}{1-\nu} \frac{(1-\underline{p})^{2}}{1-\bar{p}} \Delta \theta .
$$

Equation (23), that determines $\underline{\alpha}$, becomes

$$
V_{\underline{\alpha}}^{\prime}(\underline{\alpha}, 0)=(1-\delta) \underline{W}\left(\underline{q}^{F B}\right)-\delta \underline{\alpha}\left(-\frac{1}{S^{\prime \prime}\left(\bar{q}_{2}^{n}\right)}\right) \frac{\nu}{1-\nu} \frac{(1-\underline{p})^{2}}{1-\bar{p}}(\Delta \theta)^{2}=0 .
$$

If (27) does not have solutions (as, for instance, for $\underline{p}$ close to 1 ), then the maximum of $V(\underline{\alpha}, 0)$ is reached at $\underline{\alpha}=1$.

In the rest of this section we suppose that (27) has a solution (denoted $\underline{\alpha}(p, \bar{p})$, sometimes one or both arguments are omitted) and study its properties. We compute its derivatives $\underline{\alpha}_{\underline{p}}^{\prime}$ and $\underline{\alpha}_{\bar{p}}^{\prime}$ and look for their sign (we will use that their signs equal to the ones of $V_{\underline{\alpha} \underline{p}}^{\prime \prime}$ and $V_{\underline{\alpha} \bar{p}}^{\prime \prime}$, respectively). Then we can draw qualitatively the graph of $\underline{\alpha}(\underline{p}, \bar{p})$.

Unconditional signal Let us start with a simple case when $\underline{p}$ and $\bar{p}$ change together in the way such that $\frac{1-\underline{p}}{1-\bar{p}}$ is constant. For example, the signal is unconditional, i.e., $\underline{p}=\bar{p}=p$. Then, the second-period high cost type quantity when the type is not revealed $\bar{q}_{2}^{n}$ does not depend on the probability $p$. The only effect of an increase in $p$ is through a change in the distribution in the expectation $E_{l}\left[\bar{q}_{2 \underline{\alpha}}^{i \prime}\right]$ :

$$
V_{\underline{\alpha}}^{\prime \prime}(\underline{\alpha}, 0)=\delta \underline{\alpha} \Delta \theta \bar{q}_{2 \underline{\alpha}}^{n \prime}>0 \text {. }
$$

The sign of $V_{\underline{\alpha} p}^{\prime \prime}$ is the same as the one of $\underline{\alpha}_{p}^{\prime}$. A higher $p$ which is an improvement of the signal increases the likelihood of production in the first period, i.e., it decreases delay. The expected rent of the low cost (its derivative with respect to $\underline{\alpha}$ ) decreases so the principal prefers to have more production in the first period.

Conditional signal Now we consider the general case when $\underline{p}$ and $\bar{p}$ change independently. First, analyze the effect of an increase in $\underline{p}$. Compute $V_{\underline{\alpha} \underline{p}}^{\prime \prime}$ from (27)

$$
V_{\underline{\alpha} \underline{p}}^{\prime \prime}(\underline{\alpha}, 0)=\delta \underline{\alpha}(\Delta \theta)^{2} \frac{\nu}{1-\nu}\left(-\frac{1}{S^{\prime \prime}\left(\bar{q}_{2}^{n}\right)}\right) \frac{1-\underline{p}}{1-\bar{p}}\left[2-(1-\underline{\alpha}) \frac{1-\underline{p}}{1-\bar{p}} \Phi\left(\bar{q}_{2}^{n}\right)\right],
$$

where

$$
\Phi(q) \equiv \frac{S^{\prime \prime \prime}(q)}{\left[S^{\prime \prime}(q)\right]^{2}} \frac{\nu}{1-\nu} \Delta \theta .
$$

The sign of $V_{\underline{\alpha} \underline{p}}^{\prime \prime}$ is the same as the one of $\underline{\alpha}_{\underline{p}}^{\prime}$. The term $\Phi(q)$ is positive given Assumption 2. Then, $\underline{\alpha}_{\underline{p}}^{\prime}$ is equal to 0 if and only if the term in square brackets in (28) is equal to 0 . Denote

$$
x \equiv(1-\underline{\alpha}) \frac{1-\underline{p}}{1-\bar{p}} \frac{\nu}{1-\nu} \Delta \theta .
$$


(25) is now

$$
S^{\prime}\left(\bar{q}_{2}^{n}\right)=\bar{\theta}+x
$$

which means that $\bar{q}_{2}^{n}, S\left(\bar{q}_{2}^{n}\right)$ and all its derivatives are functions of $\bar{\theta}+x$. The term in square brackets on the right hand side of (28) is zero if and only if

$$
\frac{S^{\prime \prime \prime}\left(\bar{q}_{2}^{n}(\bar{\theta}+x)\right)}{\left[S^{\prime \prime}\left(\bar{q}_{2}^{n}(\bar{\theta}+x)\right)\right]^{2}} x=2 .
$$

If this equation has solutions $\left\{x_{i}\right\}$, then the set $\left\{\underline{\alpha}, \underline{p}, \bar{p} \mid \underline{\alpha}_{\underline{p}}^{\prime}=0\right\}$ is the union of sets defined by

$$
(1-\underline{\alpha}) \frac{1-\underline{p}}{1-\bar{p}}=\frac{(1-\nu)}{\Delta \theta \nu} x_{i}
$$

Fix a particular $\bar{p}$. In Figure 1 a possible behavior of $\underline{\alpha}(\underline{p})$ is plotted. We draw sets $\left\{\underline{\alpha}, \underline{p} \mid \underline{\alpha}_{\underline{p}}^{\prime}=0\right\}$ for some given $\bar{p}$. The sign of $\underline{\alpha}_{\underline{p}}^{\prime}$ can be determined as follows: inspecting (28) we see that for $\underline{\alpha}$ and $\underline{p}$ close to 1 this derivative is positive. In neighboring regions signs are always different. Then we draw $\underline{\alpha}(\underline{p})$ rather arbitrarily since its behavior is not very clear in the general case. There are just few sure features: first, $\underline{\alpha}(\underline{p}=0)>0$; second, $\underline{\alpha}(\underline{p})=1$ for $\underline{p}$ close to 1 ; third, $\underline{\alpha}(\underline{p})$ cannot enter a "+"-region from a "--"-region above.

Let us now consider a change in the probability of the perfect realization if the type is high cost $\bar{p}$. Take the derivative of $(27)$ :

$$
V_{\underline{\alpha} \bar{p}}^{\prime \prime}(\underline{\alpha}, 0)=\delta \underline{\alpha}(\Delta \theta)^{2} \frac{\nu}{1-\nu} \frac{1}{S^{\prime \prime}\left(\bar{q}_{2}^{n}\right)} \frac{(1-\underline{p})^{2}}{(1-\bar{p})^{2}}\left[1-(1-\underline{\alpha}) \frac{1-\underline{p}}{1-\bar{p}} \Phi\left(\bar{q}_{2}^{n}\right)\right] .
$$

The term in square brackets on the right hand side is close to the one in (28). The equation (31) will have 1 instead of 2 on the right hand side:

$$
\frac{S^{\prime \prime \prime}\left(\bar{q}_{2}^{n}(\bar{\theta}+x)\right)}{\left[S^{\prime \prime}\left(\bar{q}_{2}^{n}(\bar{\theta}+x)\right)\right]^{2}} x=1 .
$$

We can therefore conduct an analysis similar to the one of the effect of $\underline{p}$.

Proposition 11 For the signal (3) the delay may be non-monotonic in the probabilities $\underline{p}$ and $\bar{p}$.

In the next two sections I consider two particular surplus functions $S(q)$. First, I take a quadratic surplus function. Its third derivative is zero and so the delay is monotonic in $\underline{p}$ and $\bar{p}$ in the same way as in the "0-1" model. Second, I study equilibria for surplus functions such that (31) and (32) have a unique solution each. Then, the partial derivative of $\underline{\alpha}$ with respect to either $\underline{p}$ or $\bar{p}$ changes sign only once. This is the case, for example, when $S(q)=2 \sqrt{q}$. 

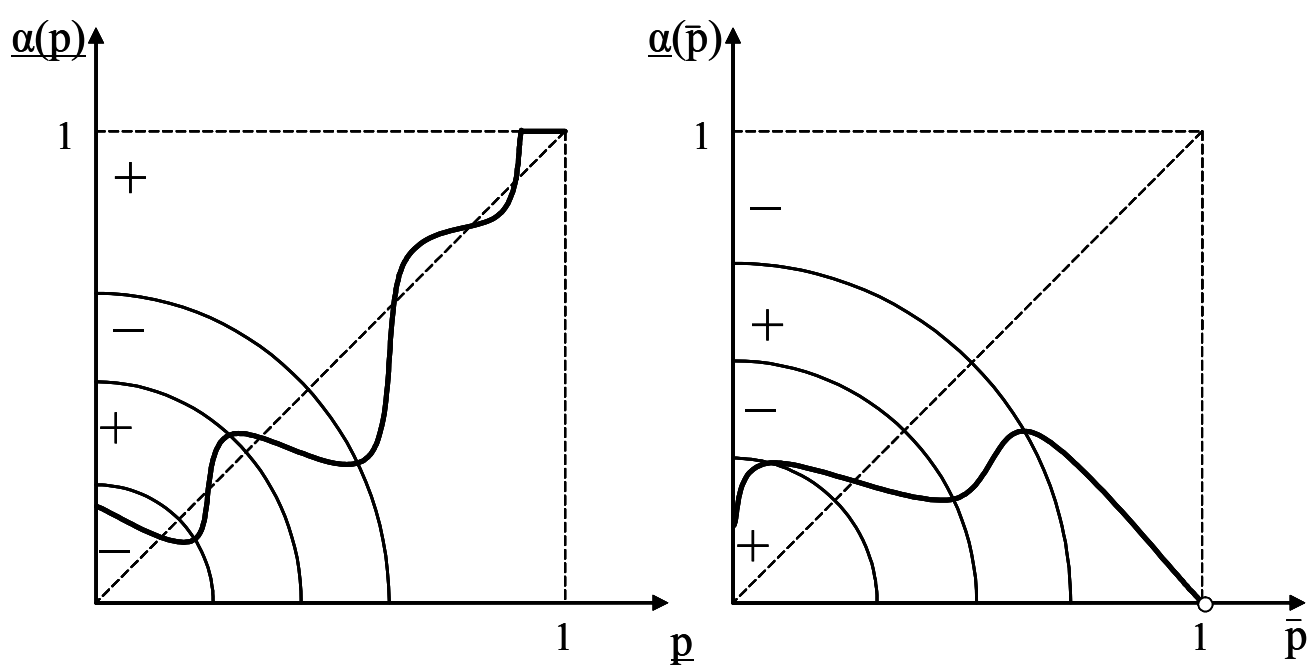

Figure 1: Possible behavior of $\underline{\alpha}(\underline{p})$ given some $\bar{p}$ (left) and of $\underline{\alpha}(\bar{p})$ given some $\underline{p}$ when (31) and (32) have three solutions each ("+" ("-") denotes regions where the derivative increases (decreases)).

\subsubsection{Delay when $\boldsymbol{S}(\boldsymbol{q})=-\frac{m}{2} \boldsymbol{q}^{2}+\boldsymbol{n} \boldsymbol{q}$}

Let us take a quadratic surplus function ${ }^{21}$

$$
S(q)=-\frac{m}{2} q^{2}+n q
$$

The first-order condition (27) becomes

$$
\frac{1-\delta}{\delta} m \frac{1-\nu}{\nu} \frac{\underline{W}\left(\underline{q}^{F B}\right)}{(\Delta \theta)^{2}}=\underline{\alpha} \frac{(1-\underline{p})^{2}}{1-\bar{p}} .
$$

The solution of this equation $\underline{\alpha}(\underline{p}, \bar{p})$ is strictly increasing in $\underline{p}$ and strictly decreasing in $\bar{p}$, i.e., the delay is decreasing in $\bar{p}$ and increasing in $\underline{p}$. This is exactly as in "0-1" model (Proposition 7).

\subsubsection{Delay when $\boldsymbol{S}(\boldsymbol{q})=2 \sqrt{q}$}

Consider now another surplus function

$$
S(q)=2 \sqrt{q} .
$$

\footnotetext{
${ }^{21} m, n>0$. We put the free term equal zero to have $S(0)=0$. We also need $n>\underline{\theta}$ to be able to find $q^{F B}$ (which is the maximum possible $q$ ). As $S^{\prime}(0)$ is finite, for realizations that are in favor of the low cost type (high $\frac{f_{l}(i)}{f_{h}(i)}$ ) the inefficient type does not produce. We disregard this assuming that probability of these events is small enough $(1-\nu$ is high enough).
} 


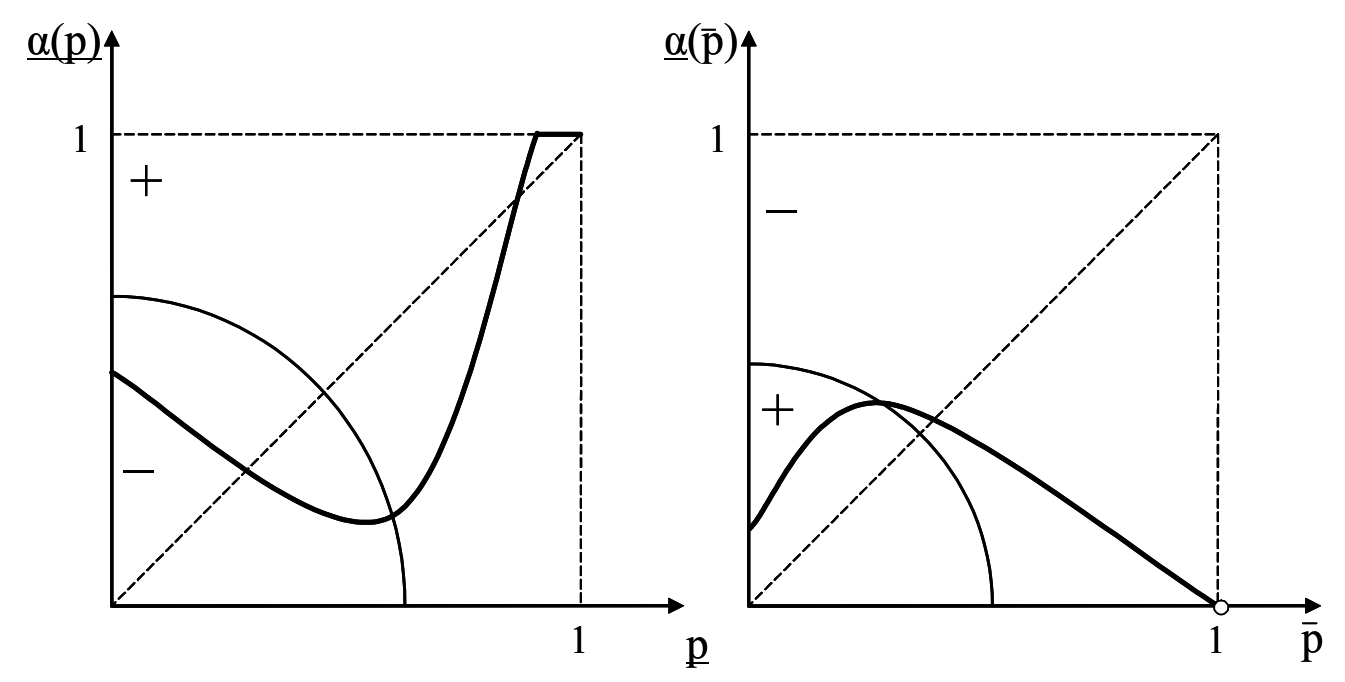

Figure 2: Possible behavior of $\underline{\alpha}(\underline{p})$ given some $\bar{p}$ (left) and of $\underline{\alpha}(\bar{p})$ given some $\underline{p}$ when (31) and (32) have a unique solution each ("+" ("-") denotes regions where the derivative increases (decreases)).

This function is concave, satisfies the Inada conditions and its third derivative is positive. Equation (31) has at most one solution $x=2 \bar{\theta}$ and (32) has at most one solution $x=\frac{\bar{\theta}}{2}$. A possible behavior of $\underline{\alpha}(\underline{p})$ and $\underline{\alpha}(\bar{p})$ is plotted in Figure 2. In fact, qualitatively these graphs are the same for any surplus function $S(q)$ such that (31) and (32) have at most one solution.

\section{Conclusion}

This paper addresses the issue of the effects of exogenous information on contracting in a dynamic agency model. The main focus of analysis has been the timing of the production, in other words, the delay in the production. Even in the simplest setting, a better information may either increase or decrease delay, depending on in which way the improvement is introduced. This is an effect of the agency relationship, as the today's costs of contracting depend on the agent's tomorrow rent (as his outside option) and they decrease with the quality of information. The effect on delay is thus unclear as tomorrow's contracting also becomes more efficient.

The paper also highlights important differences between settings where the contracted quantity can be only of a given size (the "0-1" model) and the ones where it can vary (the "variable quantity" model). In particular, when the quantity is variable, there is no delay in the benchmark case of no signals; the whole distribution of the signal matters for the 
delay; and any change in the distribution may affect the delay in a non-monotonic way. In the "0-1" model all these results are reversed.

The avenue for further research seems straightforward, though, difficult. Is it possible to provide some general conditions for the distribution of the signal such that the delay unambiguously increases (decreases)? When precisely is the delay non-monotonic in the information? How does the analysis extend to more than two types and two periods?

\section{Appendix}

\section{A The role of Assumption 1}

Take a signal with a discrete support. Any realization has a strictly positive probability. The case $\left(\underline{\alpha}=1, f_{h}(i)=0\right)$ is the most important. The expected rent of the low cost type (the term $\Delta \theta E_{l}\left[\bar{q}_{2}^{i}\right]$ in (16)) can be written as

$$
\underline{U}_{2} \equiv E_{l}\left[\underline{U}_{2}^{i}\right]=\Delta \theta E_{l}\left[\bar{q}_{2}^{i}\right]=\Delta \theta\left(\sum_{i: f_{h}(i)>0} f_{l}(i) \bar{q}_{2}^{i}+\sum_{i: f_{h}(i)=0} f_{l}(i) \bar{q}_{2}^{i}\right)=\Delta \theta \sum_{i: f_{h}(i)>0} f_{l}(i) \bar{q}_{2}^{i} .
$$

If $\underline{\alpha}=1$, in the second period there is the first-best contracting with the high cost type on the equilibrium path whenever $f_{h}(i)>0$. Therefore, when $\underline{\alpha}=1 \underline{U}_{2}$ is

$$
\left.\underline{U}_{2}\right|_{\underline{\alpha}=1}=\Delta \theta\left(\sum_{i: f_{h}(i)>0} f_{l}(i) \bar{q}^{F B}+\sum_{i: f_{h}(i)=0} f_{l}(i) \bar{q}_{2}^{i}\right)=\Delta \theta \bar{q}^{F B} \sum_{i: f_{h}(i)>0} f_{l}(i) .
$$

This rent is the outside option for the low cost type in the first-period contracting. Assumption 1 says that the second part of $\left.\underline{U}_{2}\right|_{\underline{\alpha}=1}$ which is $\Delta \theta \sum_{i: f_{h}(i)=0} f_{l}(i) \bar{q}_{2}^{i}$ is equal to 0 . It implies that this rent is the least possible which makes the strategy $\underline{\alpha}=1$ more attractive for the principal.

Note that Assumption 1 makes the profit function continuous with respect to $\underline{\alpha}$. Indeed, when $f_{h}(i)=0$ and/or $\bar{\alpha}=1$ for $\underline{\alpha}<1$ the principal proposes first-best contract to the low cost agent in the second period. Assumption 1 says that the same happens when $\underline{\alpha}=1$.

However, there is discontinuity with respect to $f_{h}(i)$. Consider the following example. Consider a signal that has two possible realizations of the following form:

$$
\left(\begin{array}{cc}
1-\varepsilon & \varepsilon \\
\varepsilon & 1-\varepsilon
\end{array}\right)
$$


If $(\underline{\alpha}, \bar{\alpha})=(1,0)$ is played the low cost type has an outside option of $\underline{U}_{2}=\Delta \theta \bar{q}^{F B}$ for $0<\varepsilon<1$. But if $\varepsilon$ is zero or one then $\underline{U}_{2}=0$.

In the same way, the rent $\underline{U}_{2}$ is not continuous in $\bar{\alpha}$ at $\bar{\alpha}=\underline{\alpha}=1$ : for $\bar{\alpha}<1 \bar{q}_{2}^{i}=\bar{q}^{F B}$ but for $\bar{\alpha}=1 \bar{q}_{2}^{i}=0$.

\section{B Proofs}

Proof of Lemma 5. If $\bar{\alpha}=1$ the refusal of the first-period contract is off equilibrium. Assumption 1 says that $\bar{q}_{2}$ is zero in this case (i.e., no rent for the low cost type). Therefore, in the first-period the low cost type accepts the contract with any positive rent. The principal's problem is then (18) without the last constraint; therefore, it is the standard adverse selection problem with probabilities $\nu$ and $(1-\nu) \bar{\alpha}$ of the low cost and the high cost type, respectively.

If $0<\bar{\alpha}<1$ the high cost type receives the first-best offer in the second period. By refusing the first-period contract the low cost agent will get an expected utility of $\delta \Delta \theta \bar{q}^{F B}$. The principal's problem is then (18) with the last constraint replaced by (20). If this constraint does not bind, we have the usual adverse selection problem. If it binds, $\underline{U}_{1}=\delta \Delta \theta \bar{q}^{F B}$ and then $\bar{q}_{1}=\delta \bar{q}^{F B}$.

Proof of Lemma 6. Take $\underline{\alpha}=\bar{\alpha}=0$. The menu specified in Lemma 4 remains optimal as (1) no type wants to deviate; (2) the principal's payoff does not depend on the menu once agent does not deviate.

Take $0<\underline{\alpha}$ and $\bar{\alpha}=0$. The menus specified in Lemma 4 and 5 remain optimal as (1) no type wants to deviate; (2) the principal's payoff from the low cost agent is maximized.

Proof of Proposition 9. Rewrite the profit function (22) as function of $\bar{\alpha}$ and $\lambda=\frac{1-\underline{\alpha}}{1-\overline{\bar{\alpha}}}:$

$$
\begin{array}{rlr}
\widetilde{V}(\lambda, \bar{\alpha})= & \nu \underline{W}\left(\underline{q}^{F B}\right)(1-\lambda(1-\bar{\alpha})+\delta \lambda(1-\bar{\alpha})) & \widetilde{V}_{1} \\
& -\nu \Delta \theta \bar{q}_{1} & \widetilde{V}_{2} \\
& +(1-\nu) \bar{\alpha} \bar{W}\left(\bar{q}_{1}\right) & \widetilde{V}_{3} \\
& +(1-\nu)(1-\bar{\alpha}) \delta E_{h}\left[\bar{W}\left(\bar{q}_{2}^{i}\right)\right] &
\end{array}
$$

$(\underline{\alpha}$ is replaced with $1-\lambda(1-\bar{\alpha}))$.

$\bar{q}_{2}$ and thus $\bar{q}_{1}$ depend only on $\lambda$, therefore $\bar{\alpha}$ enters linearly this function.

To have the maximum of $\widetilde{V}(\lambda, \bar{\alpha})$ in both mixed strategies we need $\widetilde{V}_{\bar{\alpha}}^{\prime}=\widetilde{V}_{\lambda}^{\prime}=0$. Moreover, analysis of the second-order condition 


$$
\left|\begin{array}{cc}
\widetilde{V}_{\lambda \lambda}^{\prime \prime} & \widetilde{V}_{\lambda \bar{\alpha}}^{\prime \prime} \\
\widetilde{V}_{\lambda \bar{\alpha}}^{\prime \prime} & 0
\end{array}\right|=-\left(\widetilde{V}_{\lambda \bar{\alpha}}^{\prime \prime}\right)^{2} \leq 0
$$

shows that there also must be $\widetilde{V}_{\lambda \bar{\alpha}}^{\prime \prime}=0 .{ }^{22}$

Let us find the first-order condition for $\lambda$ :

$$
\begin{aligned}
\widetilde{V}_{\lambda}^{\prime}=-\nu \underline{W}\left(\underline{q}^{F B}\right)(1-\bar{\alpha})(1-\delta)-\nu \Delta \theta \bar{q}_{1 \lambda}^{\prime}+ & (1-\nu) \bar{\alpha}\left(S^{\prime}\left(\bar{q}_{1}\right)-\bar{\theta}\right) \bar{q}_{1 \lambda}^{\prime} \\
& +(1-\nu)(1-\bar{\alpha}) \delta \frac{\partial}{\partial \lambda}\left[E_{h}\left[\bar{W}\left(\bar{q}_{2}^{i}\right)\right]\right]=0
\end{aligned}
$$

Rewrite it as

$$
\begin{gathered}
\bar{\alpha}\left\{\nu \underline{W}\left(\underline{q}^{F B}\right)(1-\delta)+(1-\nu)\left(S^{\prime}\left(\bar{q}_{1}\right)-\bar{\theta}\right) \bar{q}_{1 \lambda}^{\prime}-(1-\nu) \delta \frac{\partial}{\partial \lambda}\left[E_{h}\left[\bar{W}\left(\bar{q}_{2}^{i}\right)\right]\right]\right\}= \\
\nu \underline{W}\left(\underline{q}^{F B}\right)(1-\delta)+\nu \Delta \theta \bar{q}_{1 \lambda}^{\prime}-(1-\nu) \delta \frac{\partial}{\partial \lambda}\left[E_{h}\left[\bar{W}\left(\bar{q}_{2}^{i}\right)\right]\right]
\end{gathered}
$$

The expression in the brackets in the left-hand side is exactly $\widetilde{V}_{\lambda \bar{\alpha}}^{\prime \prime}$. So, at the point when $\widetilde{V}_{\lambda \bar{\alpha}}^{\prime \prime}=0$ the right-hand must also be zero. Summing up the last two terms of the right-hand side we obtain:

$$
\begin{aligned}
& \nu \Delta \theta \bar{q}_{1 \lambda}^{\prime}-(1-\nu) \delta E_{h}\left[\left(S^{\prime}\left(\bar{q}_{2}^{i}\right)-\bar{\theta}\right) \bar{q}_{2 \lambda}^{i \prime}\right]= \\
& \delta \nu \Delta \theta E_{l}\left[\bar{q}_{2 \lambda}^{i \prime}\right]-\delta(1-\nu) \int_{0}^{+\infty} \frac{\nu}{1-\nu} \lambda \frac{f_{l}(i)}{f_{h}(i)} \Delta \theta \bar{q}_{2 \lambda}^{i \prime} f_{h}(i) d i= \\
& \delta \nu \Delta \theta E_{l}\left[\bar{q}_{2 \lambda}^{i \prime}\right]-\delta \nu \lambda \Delta \theta \int_{0}^{+\infty} \bar{q}_{2 \lambda}^{i \prime} f_{l}(i) d i= \\
& \delta \nu \Delta \theta E_{l}\left[\bar{q}_{2 \lambda}^{i \prime}\right](1-\lambda) .
\end{aligned}
$$

(we used $\bar{q}_{1 \lambda}^{\prime}=\delta E_{l}\left[\bar{q}_{2 \lambda}^{i \prime}\right]$ from Lemma 4 and $S^{\prime}\left(\bar{q}_{2}^{i}\right)-\bar{\theta}=\frac{\nu}{1-\nu} \lambda \frac{f_{l}(i)}{f_{h}(i)} \Delta \theta$ from (13)).

Examining (33) we find that if both sides are equal to zero, then

$$
(1-\nu)\left(S^{\prime}\left(\bar{q}_{1}\right)-\bar{\theta}\right)=\nu \Delta \theta
$$

provided that $\bar{q}_{1 \lambda}^{\prime}$ is different from zero. Indeed, if $\bar{q}_{1 \lambda}^{\prime}=0$ then $\bar{q}_{2 \lambda}^{i \prime}=0 \forall i$ but then the right-hand side of $(33)$ is positive: $\nu \underline{W}\left(\underline{q}^{F B}\right)(1-\delta)>0$. So, $\bar{q}_{1 \lambda}^{\prime} \neq 0$. We can conclude that

${ }^{22} \widetilde{V}_{\lambda \lambda}^{\prime \prime}$ is linear in $\bar{\alpha}$, so the second-order condition with respect to $\lambda$ can be written as:

$$
\left\{\begin{array}{l}
\widetilde{V}_{\lambda \lambda}^{\prime \prime} \mid \bar{\alpha}=0=\delta\left\{(1-\nu) E_{b}\left[\left(\bar{q}_{2 \lambda}^{\prime}\right)^{2} S^{\prime \prime}\left(\bar{q}_{2}\right)\right]-E_{g}\left[\bar{q}_{2 \lambda \lambda}^{\prime \prime}\right](1-\lambda) \nu \Delta \theta\right\}<0, \\
\widetilde{V}_{\lambda \lambda}^{\prime \prime} \mid \bar{\alpha}=1=\delta^{2}(1-\nu) S^{\prime \prime}\left(\bar{q}_{1}\right)\left(E_{g}\left[\bar{q}_{2 \lambda}^{\prime}\right]\right)^{2}<0 .
\end{array}\right.
$$

$\left.\widetilde{V}_{\lambda \lambda}^{\prime \prime}\right|_{\bar{\alpha}=0}$ is negative if $S^{\prime \prime \prime}(q)$ is non-negative and $\left.\widetilde{V}_{\lambda \lambda}^{\prime \prime}\right|_{\bar{\alpha}=1}$ is always negative. (We would like to have this second-order condition unsatisfied as then we don't need to prove anything further). 
$\bar{q}_{1}$ must be equal to the usual second-best quantity $\bar{q}^{S B}$ with probabilities $\nu$ and $(1-\nu)$ of the low cost and the high cost type, respectively (it is given by $(21)$ with $\bar{\alpha}=1$ ).

To sum up what we did until now, an interior solution must satisfy the following three conditions:

$$
\left\{\begin{array}{l}
\frac{\nu}{1-\nu} \underline{W}\left(\underline{q}^{F B}\right)(1-\delta) \lambda+\bar{W}\left(\bar{q}_{1}\right)=\delta E_{h}\left[\bar{W}\left(\bar{q}_{2}^{i}\right)\right] \\
\delta E_{l}\left[\bar{q}_{2}^{i}\right]=\bar{q}^{S B} \\
\underline{W}\left(\underline{q}^{F B}\right)(1-\delta)=-\delta \Delta \theta E_{l}\left[\bar{q}_{2 \lambda}^{i \prime}\right](1-\lambda) .
\end{array}\right.
$$

The first condition is that $\widetilde{V}_{\bar{\alpha}}^{\prime}=0$ and the two others are the consequences of $\widetilde{V}_{\lambda \bar{\alpha}}^{\prime \prime}=$ $\widetilde{V}_{\lambda}^{\prime}=0$.

I could not prove directly that these conditions are not compatible. Instead, I differentiate the first two with respect to $\underline{\theta}$ and use the third one to show that they are compatible only if $\lambda=0$.

First, differentiate $E_{l}\left[\bar{q}_{2}^{i i}\right]$ :

$E_{l}\left[\bar{q}_{2 \underline{\theta}}^{i \prime}\right]=E_{l}\left[\frac{d}{d \underline{\theta}} \bar{q}_{2}^{i}\right]=E_{l}\left[\bar{q}_{2 \lambda}^{i \prime} \lambda_{\underline{\theta}}^{\prime}+\frac{\partial}{\partial \underline{\theta}} \bar{q}_{2}^{i}\right]=E_{l}\left[\bar{q}_{2 \lambda}^{i \prime} \lambda_{\underline{\theta}}^{\prime}-\frac{1}{S^{\prime \prime}\left(\bar{q}_{2}^{i}\right)} \frac{\nu}{1-\nu} \lambda \frac{f_{l}(i)}{f_{h}(i)}\right]=\left(\lambda_{\underline{\theta}}^{\prime}-\frac{\lambda}{\Delta \theta}\right) E_{l}\left[\bar{q}_{2 \lambda}^{i \prime}\right]$.

So,

$$
\left(\bar{q}^{S B}\right)_{\underline{\theta}}^{\prime}=\delta\left(\lambda_{\underline{\theta}}^{\prime}-\frac{\lambda}{\Delta \theta}\right) E_{l}\left[\bar{q}_{2 \lambda}^{i \prime}\right] .
$$

Second, differentiate the first condition:

$$
\begin{array}{r}
\frac{\nu}{1-\nu}\left(S^{\prime}\left(\underline{q}^{F B}\right)-\bar{\theta}\right)\left(\underline{q}^{F B}\right)_{\underline{\theta}}^{\prime}(1-\delta) \lambda+\frac{\nu}{1-\nu} \underline{W}\left(\underline{q}^{F B}\right)(1-\delta) \lambda_{\underline{\theta}}^{\prime}+\left(S^{\prime}\left(\bar{q}^{S B}\right)-\bar{\theta}\right)\left(\bar{q}^{S B}\right)_{\underline{\theta}}^{\prime}= \\
\delta E_{h}\left[\left(S^{\prime}\left(\bar{q}_{2}^{i}\right)-\bar{\theta}\right) \bar{q}_{2 \underline{\theta}}^{i \prime}\right] .
\end{array}
$$

Noting that $S^{\prime}\left(\underline{q}^{F B}\right)-\bar{\theta}=0, S^{\prime}\left(\bar{q}^{S B}\right)-\bar{\theta}=\frac{\nu}{1-\nu} \Delta \theta$ and $E_{h}\left[\left(S^{\prime}\left(\bar{q}_{2}^{i}\right)-\bar{\theta}\right) \bar{q}_{2 \underline{\theta}}^{i}\right]=$ $\frac{\nu}{1-\nu} \lambda \Delta \theta E_{l}\left[\bar{q}_{2 \underline{\theta}}^{i \prime}\right]$ we get

$$
\underline{W}\left(\underline{q}^{F B}\right)(1-\delta) \lambda_{\underline{\theta}}^{\prime}+\Delta \theta\left(\bar{q}^{S B}\right)_{\underline{\theta}}^{\prime}=\delta \Delta \theta \lambda E_{l}\left[\bar{q}_{2 \underline{\theta}}^{i \prime}\right] .
$$

Using (36)

$$
\underline{W}\left(\underline{q}^{F B}\right)(1-\delta) \lambda_{\underline{\theta}}^{\prime}+\Delta \theta(1-\lambda) \delta E_{l}\left[\bar{q}_{2 \lambda}^{i \prime}\right]\left(\lambda_{\underline{\theta}}^{\prime}-\frac{\lambda}{\Delta \theta}\right)=0
$$

Third, use the third condition from (35) that $\Delta \theta(1-\lambda) \delta E_{l}\left[\bar{q}_{2 \lambda}^{i \prime}\right]=-\underline{W}\left(\underline{q}^{F B}\right)(1-\delta)$ which results in

$$
\lambda=0 .
$$


But this means that $\underline{\alpha}$ is equal to 1 .

Remark 5. When $\lambda=0$ the first two conditions of (35) are not compatible: the second one becomes $\delta \bar{q}^{F B}=\bar{q}^{S B}$ (which can be true only non-generically) but then the first one is not satisfied as $\bar{W}\left(\delta \bar{q}^{F B}\right)>\delta \bar{W}\left(\bar{q}^{F B}\right)$.

Proof of Proposition 10. By Proposition 9 the maximum of the principal's payoff is not reached when both types play a mixed strategy. So, it must be reached in at least one of the following four cases: ${ }^{23}$

1. $(\underline{\alpha}, \bar{\alpha})=(\underline{\alpha}, 1)$. In the second period the principal is sure to face the low cost agent. Then she proposes him a contract with no rent. Any first-period offer that high cost agent accepts gives some rent to the low cost type, so he will always accept the first-period offer. Thus, $\underline{\alpha}=1 .^{24,25}$

2. $(\underline{\alpha}, \bar{\alpha})=(\underline{\alpha}, 0)$. The principal maximizes

$$
\begin{aligned}
V(\underline{\alpha}, 0)= & \frac{\nu W}{W}\left(\underline{q}^{F B}\right)(\underline{\alpha}+\delta(1-\underline{\alpha})) \\
& -\nu \Delta \theta \delta E_{l}\left[\bar{q}_{2}^{i}\right] \\
& +(1-\nu) \delta E_{h}\left[\bar{W}\left(\bar{q}_{2}^{i}\right)\right]
\end{aligned}
$$

which is $(22)$ for $\bar{\alpha}=0$ and $\bar{q}_{1}=\delta E_{l}\left[\bar{q}_{2}^{i}\right]$. Derivating with respect to $\underline{\alpha}$ yields

$$
V_{\underline{\alpha}}^{\prime}(\underline{\alpha}, 0)=\nu \underline{W}\left(\underline{q}^{F B}\right)(1-\delta)-\nu \delta \underline{\alpha} \Delta \theta E_{l}\left[\bar{q}_{2 \underline{\alpha}}^{i \prime}\right]
$$

which is the first-order condition stated in (23) (we can write the second term as $-\nu \delta \underline{\alpha} \Delta \theta E_{l}\left[\bar{q}_{2}^{i}\right]-\nu \delta(1-\underline{\alpha}) \Delta \theta E_{l}\left[\bar{q}_{2}^{i}\right]$. Then only $E_{l}\left[\bar{q}_{2}^{i}\right]$ in the first part should be derivated as the second one enters the second-period payoff for which $\bar{q}_{2}^{i}$ is optimal so we can apply the envelope theorem).

This derivative is positive at $\underline{\alpha}=0$ so $\underline{\alpha}>0\left(\bar{q}_{2 \underline{\alpha}}^{i \prime}\right.$ is positive).

\footnotetext{
${ }^{23} V(\underline{\alpha}, \bar{\alpha})$ is bounded by 0 and its first-best value; the set of admissible $(\underline{\alpha}, \bar{\alpha})$ is compact: $[0,1] \times[0,1]$. If $V(\underline{\alpha}, \bar{\alpha})$ is continous, it has a maximum. When it is not continuous, it still has a maximum (case 3c).

${ }^{24}$ Clearly, the inability of the principal to commit to a second period contract is crucial for nonimplementability.

${ }^{25}$ Note that for a given signal there might be some other acceptance probabilities that are not implementable. For example, if in the second period the type of the agent is always known then any $(\underline{\alpha}<1, \bar{\alpha}>0)$ is not implementable since the efficient agent never gets a rent in the second period.
} 
3. $(\underline{\alpha}, \bar{\alpha})=(1, \bar{\alpha})$. The analysis of this case depends whether the distribution of the signal is atomless or not (whether $\sum_{i: p_{i \mid h}>0} p_{i \mid l}$ is less or equal one). We give the analysis for the general case. In the end of this case we say what changes for the continuous support $\left(\sum_{i: p_{i \mid h}>0} p_{i \mid l}=1\right)$. We consider $\bar{\alpha}<1$ as $\bar{\alpha}=1$ is treated in case 1 . According to Lemma 5,

$$
\bar{q}_{1}=\max \left\{\delta \bar{q}^{F B}, \bar{q}_{1}(\bar{\alpha})\right\} \text {, where } \bar{q}_{1}(\bar{\alpha}) \text { is given by }(21) \text {. }
$$

The principal's profit function from $(22)$ is

$$
V(1, \bar{\alpha})=\nu \underline{W}\left(\underline{q}^{F B}\right)-\nu \Delta \theta \bar{q}_{1}+(1-\nu) \bar{\alpha} \bar{W}\left(\bar{q}_{1}\right)+\delta(1-\nu)(1-\bar{\alpha}) \bar{W}\left(\bar{q}^{F B}\right) .
$$

First, we establish the behavior of $\bar{q}_{1}$. From its definition we see that $\bar{q}_{1}(\bar{\alpha})$ is increasing in $\bar{\alpha}$ and $\bar{q}_{1}(0)=0$. Therefore, for $\bar{\alpha}$ small $\bar{q}_{1}(\bar{\alpha})$ is less than $\delta \bar{q}^{F B} \sum_{i: p_{i \mid h}>0} p_{i \mid l}$ and at some point it might become greater:

$$
\bar{q}_{1}=\left[\begin{array}{cc}
\delta \bar{q}^{F B} \sum_{i: p_{i \mid h}>0} p_{i \mid l}, & \text { if } \bar{\alpha}<\widehat{\bar{\alpha}} \\
\bar{q}_{1}(\bar{\alpha}) & \text { otherwise }
\end{array},\right.
$$

where $\widehat{\bar{\alpha}}$ is the solution to the equation $\bar{q}_{1}(\bar{\alpha})=\delta \bar{q}^{F B} \sum_{i: p_{i \mid h}>0} p_{i \mid l}$ (if it exists in the range $[0,1]$ ). (Note that if $\bar{\alpha}=1 \bar{q}_{1}=\bar{q}_{1}(1)$. Thus there is a breakpoint in $\bar{q}_{1}$ if $\widehat{\bar{\alpha}}$ does not exist).

Second, we find the derivative of $V(1, \bar{\alpha})$ with respect to $\bar{\alpha}$ :

$$
V_{\bar{\alpha}}^{\prime}(1, \bar{\alpha})=(1-\nu)\left[\bar{W}\left(\bar{q}_{1}\right)-\delta \bar{W}\left(\bar{q}^{F B}\right)\right] .
$$

When $\bar{q}_{1}=\delta \bar{q}^{F B} \sum_{i: p_{i \mid h}>0} p_{i \mid l}$ this derivative clearly has this form. When $\bar{q}_{1}=\bar{q}_{1}(\bar{\alpha})$ we use the envelope theorem as for a given $\bar{\alpha}$ the quantity $\bar{q}_{1}(\bar{\alpha})$ is optimal.

Since $\bar{W}\left(\bar{q}_{1}\right)$ is a strictly increasing function of $\bar{q}_{1}$ up to $\bar{q}^{F B}$, the behavior of $V_{\bar{\alpha}}^{\prime}(1, \bar{\alpha})$ is similar to the one of $\bar{q}_{1}$.

Finally, we can conclude that given the form of $\bar{W}\left(\bar{q}_{1}\right)$ there are four possible cases:

a. $\bar{W}\left(\bar{q}_{1}\right)>\delta \bar{W}\left(\bar{q}^{F B}\right)$ for any $\bar{\alpha} \in[0,1]$. Then $V_{\bar{\alpha}}^{\prime}(1, \bar{\alpha})$ is always positive. The principal implements the no-delay solution $(1,1)$.

b. $\bar{W}\left(\bar{q}_{1}\right)<\delta \bar{W}\left(\bar{q}^{F B}\right)$ for any $\bar{\alpha} \in[0,1]$. Then $V_{\bar{\alpha}}^{\prime}(1, \bar{\alpha})$ is always negative, so $\bar{\alpha}=0$. 
c. $\bar{q}_{1}(1)<\delta \bar{q}^{F B} \sum_{i: p_{i \mid h}>0} p_{i \mid l}$ and $\bar{W}\left(\delta \bar{q}^{F B} \sum_{i: p_{i \mid h}>0} p_{i \mid l}\right)>\delta \bar{W}\left(\bar{q}^{F B}\right)>\bar{W}\left(\bar{q}_{1}(1)\right)$. This is the case of a breakpoint of $\bar{W}\left(\bar{q}_{1}\right)$ at $\bar{\alpha}=1$. When $\bar{\alpha}<1$ the principal has to satisfy the $\underline{I C}_{i n t}$ constraint of the low cost type, then $\bar{q}_{1}$ is high so it is better to produce in the first period. But at $\bar{\alpha}=1 \bar{q}_{1}(1)$ is low so it is better to postpone the production. The principal wants to have $\bar{\alpha}$ as close to 1 as possible but not 1 , i.e., the maximum is not achieved in the range $[0,1)$. The principal's payoff does not exceed $\sup _{\bar{\alpha} \in[0,1)} V(1, \bar{\alpha})=\nu \underline{W}\left(\underline{q}^{F B}\right)-\nu \Delta \theta \bar{q}_{1}+(1-\nu) \bar{W}\left(\bar{q}_{1}\right)$, where $\bar{q}_{1}=\delta \bar{q}^{F B} \sum_{i: p_{i \mid h}>0} p_{i \mid l}$. But this cannot be higher than the no-delay payoff $V(1,1)=\nu \underline{W}\left(\underline{q}^{F B}\right)-\nu \Delta \theta \bar{q}_{1}(1)+(1-\nu) \bar{W}\left(\bar{q}_{1}(1)\right)$ since by definition $\bar{q}_{1}(1)$ maximizes the payoff in case of no delay.

d. There exists $\widetilde{\bar{\alpha}}$ at which $\bar{W}\left(\bar{q}_{1}(\widetilde{\bar{\alpha}})\right)=\delta \bar{W}\left(\bar{q}^{F B}\right)$. For $\bar{\alpha}<\widetilde{\bar{\alpha}} V_{\bar{\alpha}}^{\prime}$ is negative while for $\bar{\alpha}>\widetilde{\bar{\alpha}}$ it is positive. $V(1, \bar{\alpha})$ has the minimum at $\bar{\alpha}=\widetilde{\bar{\alpha}}$ and the maximum is at one of the ends, i.e., $\bar{\alpha}$ is 1 or 0 .

If $f_{h}(i)>0$ for any $i$ (for example, continuous support) the case 3 can be reinforced: if $\underline{\alpha}=1$ then $\bar{\alpha}=1$. This follows from the concavity of $\bar{W}(q)$ :

$$
\bar{W}\left(\delta \bar{q}^{F B}\right)>\delta \bar{W}\left(\bar{q}^{F B}\right) .
$$

Then the subcases $(b)$ and $(d)$ do not exist.

4. $(\underline{\alpha}, \bar{\alpha})=(0, \bar{\alpha})$. Derivating the profit function $(22)$ with respect to $\underline{\alpha}$ gives

$$
\begin{aligned}
V_{\underline{\alpha}}^{\prime}(\underline{\alpha}, \bar{\alpha})= & \nu \underline{W}\left(\underline{q}^{F B}\right)(1-\delta)-\nu \delta \underline{\alpha} \Delta \theta \\
& E_{l}\left[\bar{q}_{2 \underline{\alpha}}^{i \prime}\right]+(1-\nu) \bar{\alpha}\left(S^{\prime}\left(\bar{q}_{1}\right)-\bar{\theta}\right) E_{l}\left[\bar{q}_{2 \underline{\alpha}}^{i \prime}\right]
\end{aligned}
$$

(to get the middle term we used the same trick as in the case 2). This derivative is positive at $\underline{\alpha}=0$, thus the maximum of principal's profits is not at $\underline{\alpha}=0$.

Let us now check the second-order condition. From (23)

$$
V_{\underline{\alpha \alpha}}^{\prime \prime}=\delta\left\{(1-\nu) E_{h}\left[\left(\bar{q}_{2 \underline{\alpha}}^{i \prime}\right)^{2} S^{\prime \prime}\left(\bar{q}_{2}^{i}\right)\right]-\nu \Delta \theta \underline{\alpha} E_{l}\left[\bar{q}_{2 \underline{\alpha \alpha}}^{i \prime \prime}\right]\right\} \leq 0
$$

which is satisfied if $S^{\prime \prime \prime}(q) \geq 0$. (One can see that $\bar{q}_{2 \underline{\alpha \alpha}}^{i \prime \prime}=\frac{S^{\prime \prime \prime}\left(\bar{q}_{2}^{i}\right)}{\left[S^{\prime \prime}\left(\bar{q}_{2}^{i}\right)\right]^{2}} \bar{q}_{2 \underline{\alpha}}^{i \prime} \frac{\nu}{1-\nu} \frac{f_{l}(i)}{f_{h}(i)} \Delta \theta$ so $\bar{q}_{2 \underline{\alpha \alpha}}^{i \prime \prime}$ has the same sign as $\left.S^{\prime \prime \prime}\left(\bar{q}_{2}^{i}\right)\right)$.

As $V_{\underline{\alpha}}^{\prime}(\underline{\alpha}, 0)$ is increasing at $\underline{\alpha}=0$, when if $V(\underline{\alpha}, 0)$ is not concave, the maximum is always either the solution of $(23)$ or $\underline{\alpha}=1$. In the latter case, $\bar{\alpha}$ is also one as shown in case 3 .

Remark 6. For the discrete support note the following. Since $V(\underline{\alpha}, 0)$ is continuous in $\underline{\alpha},(1,0)$ is preferred to $(\underline{\alpha}, 0)$ if the first-order condition $(23)$ does not have solutions 
(i.e., $V(\underline{\alpha}, 0)$ is increasing for any $\underline{\alpha})$. If $V(\underline{\alpha}, 0)$ is not concave in $\underline{\alpha}$, then (23) does not give the global maximum. $V(1,0)$ should be compared directly to the value of $V(\underline{\alpha}, 0)$ at candidate solutions.

\section{References}

Bagnoli, M., S. Salant, and J. Swierzbinski (1992): "Intertemporal Self-selection with Multiple Buyers Under Complete and Incomplete Information," University of Michigan Center for Research on Economics and Social Theory (CREST) Working Paper.

(1995): "Intertemporal Self-selection with Multiple Buyers," Economic Theory, $5,513-526$.

Baron, D., and R. Myerson (1982): "Regulating a Monopolist with Unknown Costs," Econometrica, 50(4), 911-930.

Bester, H., And R. Strausz (2001): "Contracting with Imperfect Commitment and the Revelation Principle: The Single Agent Case," Econometrica, 69(4), 1077-1098.

Bolton, P., and M. Dewatripont (2005): Contract Theory. MIT Press.

Dixit, A., And R. Pindyck (1994): Investment under Uncertainty. Princeton University Press.

FudenberG, D., And J. Tirole (1983): "Sequential Bargaining with Incomplete Information," Review of Economic Studies, 50(2), 221-247.

- (1990): "Moral Hazard and Renegotiation in Agency Contracts," Econometrica, 58(6), 1279-1319.

Gul, F., H. Sonnenschein, And R. Wilson (1986): "Foundations of Dynamic Monopoly and the Coase Conjecture," Journal of Economic Theory, 39, 155-190.

Hart, O., And J. Tirole (1988): "Contract Renegotiation and Coasian Dynamics," Review of Economic Studies, 55, 509-540.

Laffont, J.-J., and D. Martimort (2002): The Theory of Incentives: The PrincipalAgent Model. Princeton University Press.

Laffont, J.-J., And J. Tirole (1987): "Comparative Statics of the Optimal Dynamic Incentive Contract," European Economic Review, 31(4), 901-926. 
— (1990): "Adverse Selection and Renegotiation in Procurement," Review of Economic Studies, 57(4), 597-625.

Sobel, J., and I. Takahashi (1983): "A Multistage Model of Bargaining," Review of Economic Studies, 50(3), 411-426.

Stokey, N. (1981): "Rational Expectations and Durable Goods Pricing," Bell Journal of Economics, 12(1), 112-128.

Wang, G. (1998): "Bargaining over a Menu of Wage Contracts," Review of Economic Studies, 65, 295-305. 